\title{
CONSTRUCTION OF MSE WALL USING TIRE SHRED-SAND MIXTURE AS BACKFILL
}

\section{SPR-3470}

SAC MEMBERS : BARRY PARTRIDGE

NAYYAR ZIA SIDDIKI

\section{Final Project Report (PPT)}

\section{Construction of MSE Wall using Tire Shred-Sand Mixture as Backfill}

\author{
SPR -3470 \\ Purdue University, West Lafayette \\ Project Investigator: Monica Prezzi \\ Masters Student: Varenya Kumar Duvvuru Mohan
}




\section{Contents}

3

Background

- Project Overview

a Construction of the MSE wall using tire shred-sand mixtures

口 Tasks

- Design Summary

\section{Background}

- Existing pavement at $\mathrm{l}-80$ needed widening

口 A tire shred-sand mixture and EPS were used as backfill for MSE wall No. 54

- The tire shred-sand mixture was designed to be lightweight to minimize vertical settlement of the MSE wall

$\square$ The geotechnical properties needed for design were determined

- The performance of the wall was monitored 


\section{Project Overview}

I-80/94 interchange modification (Wall No. 54)

- Lake County, Indiana

- Sta. 676+00 "M" to Sta. 681+00 "L"

- Wall height $=10 \mathrm{ft}$

- Backfill: tire shred-sand mixture + EPS

- Mixing ratio by volume (loose state):

- 60 (tire shred):40 (sand)

- Materials used (compacted state):

- Shredded tires: 2,000 $\mathrm{yd}^{3}$

- Sand: $1,300 \mathrm{yd}^{3}$

- No. of compactor passes $=4 \sim 5$

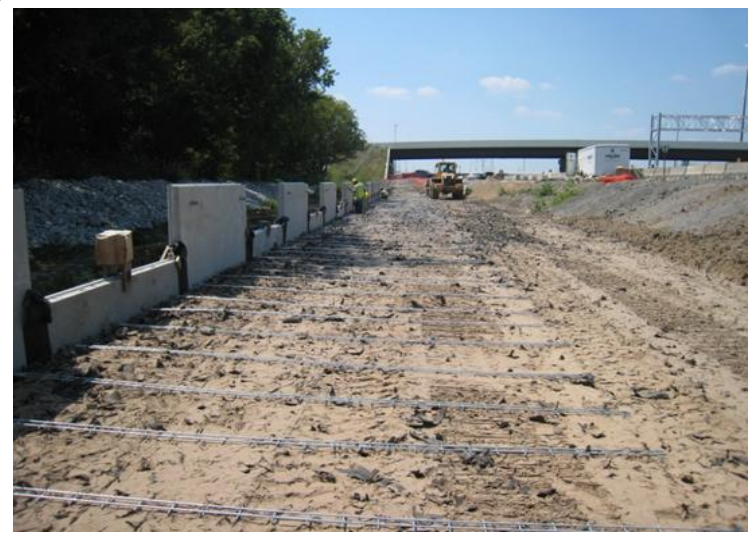

\section{Construction of MSE wall}

\section{MIXING PROCESS}

Three buckets of shredded tires

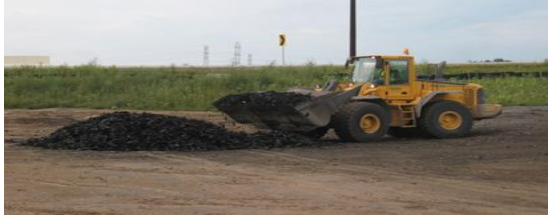

Two buckets of sands

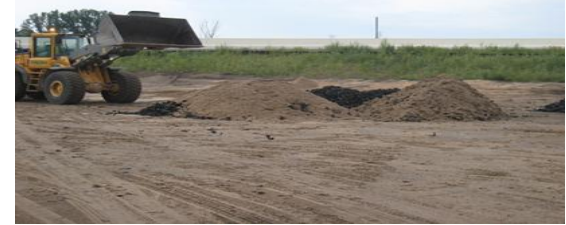

Tire shred-sand mixture

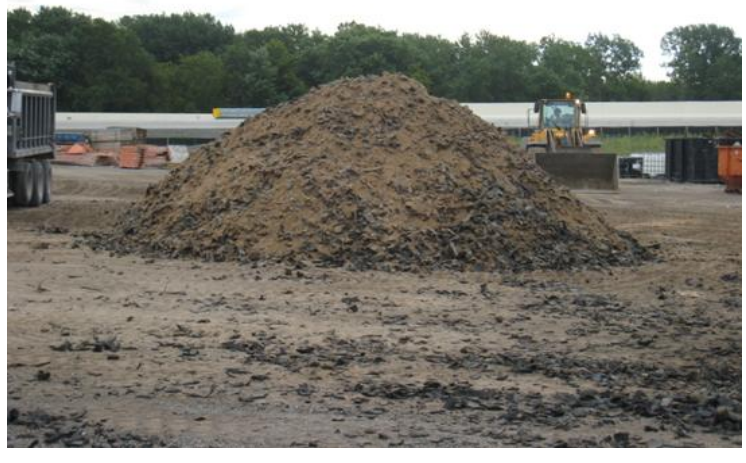




\section{Construction of MSE wall}

7

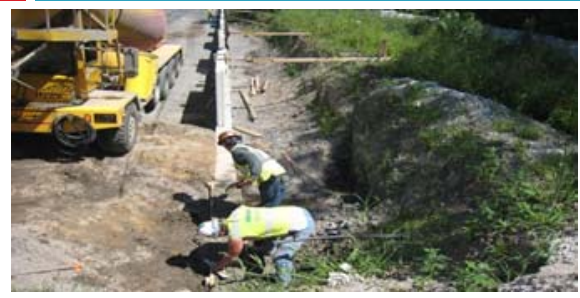

Installation of leveling pad
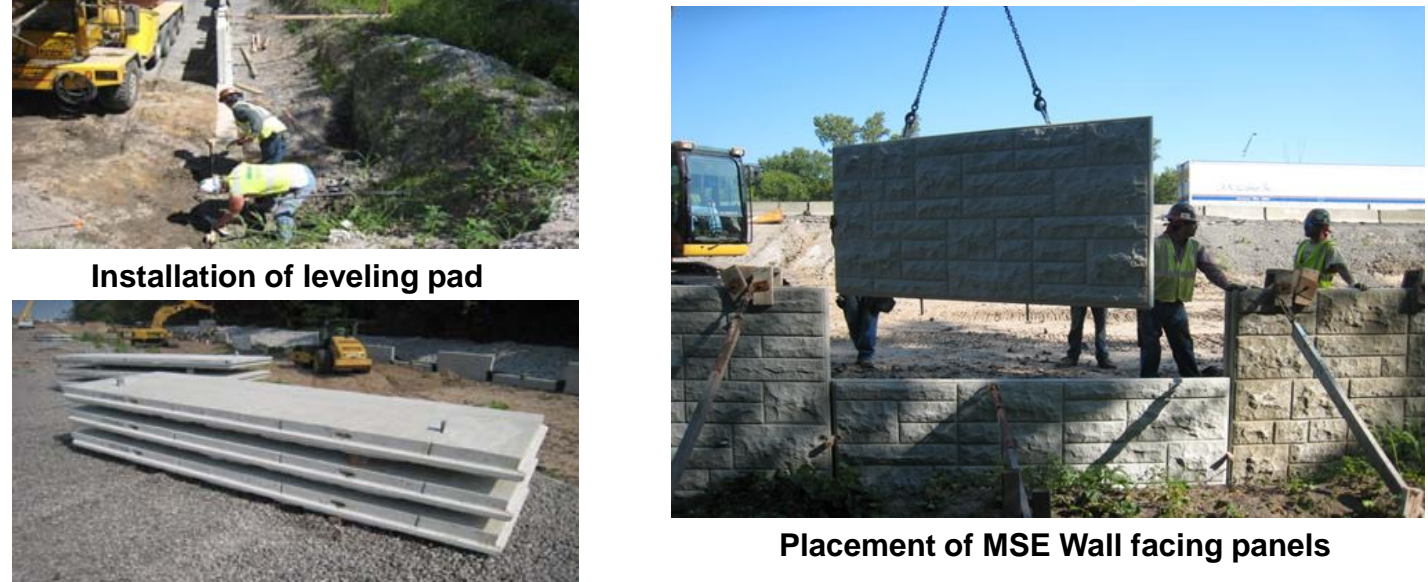

Placement of MSE Wall facing panels

Facing panels ready to be installed

\section{Construction of MSE wall}

\section{BACKFILLING}

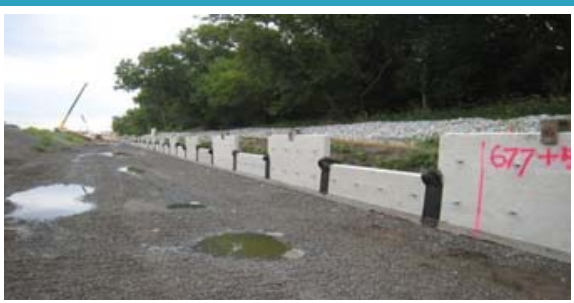

Aggregates on the bottom of the fill

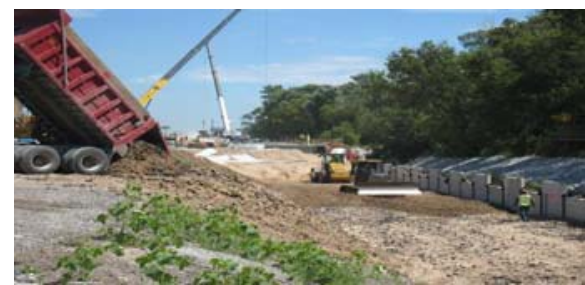

Hauling and spreading the mixture
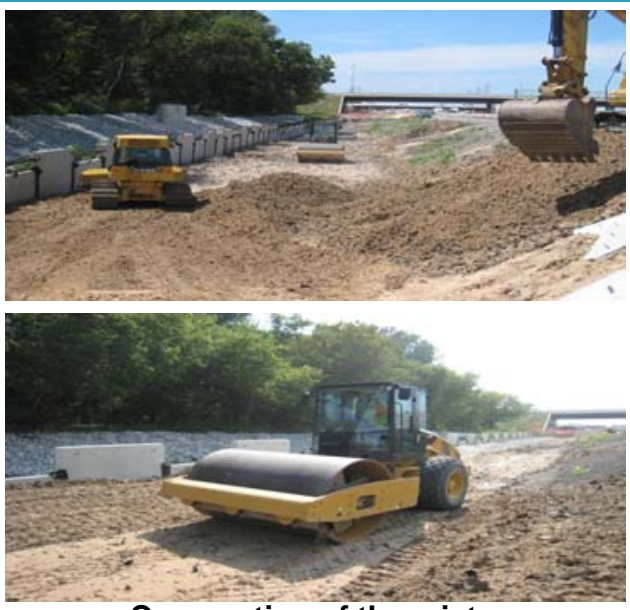

Compaction of the mixture (10-ton roller, lifts of 12 inches) 


\section{Construction of MSE wall}

9

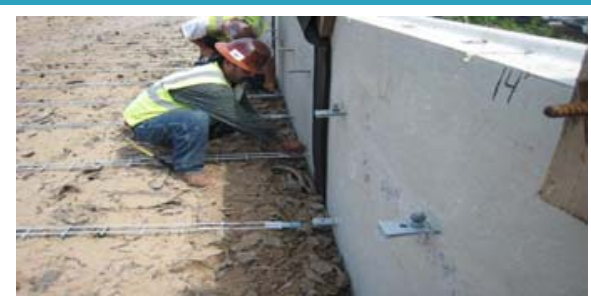

Connection of reinforcement to facing

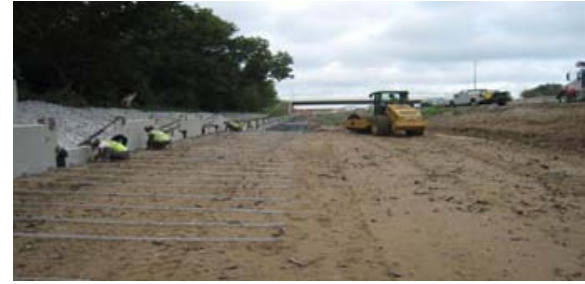

Installed reinforcements

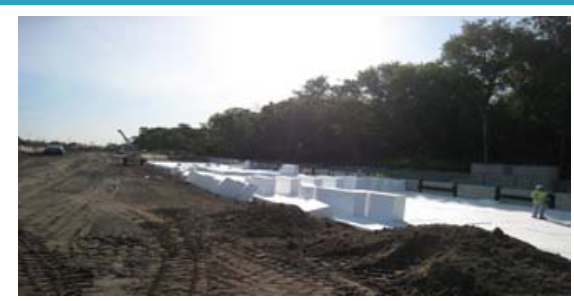

Installation of EPS blocks

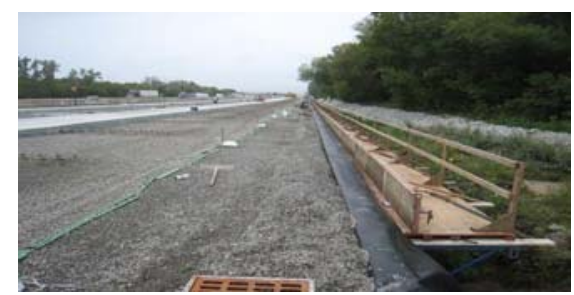

Aggregate and reinforcing bars on the EPS

\section{Tasks}

\section{Tasks in the proposal}

- Assess compaction characteristics of mixture

- Supervise MSE wall construction

- Perform $Q C$ tests during construction

- Assess performance of wall

- Prepare a power point presentation with results

\section{Additional Tasks}

- Perform lab tests at 3 different mixing ratios $-50: 50,60: 40$ and 70:30 by volume of TS : Sand

$>$ Laboratory pullout tests

$>$ Direct shear tests

- Include design summary in the ppt 


\section{Tasks - Field Monitoring}

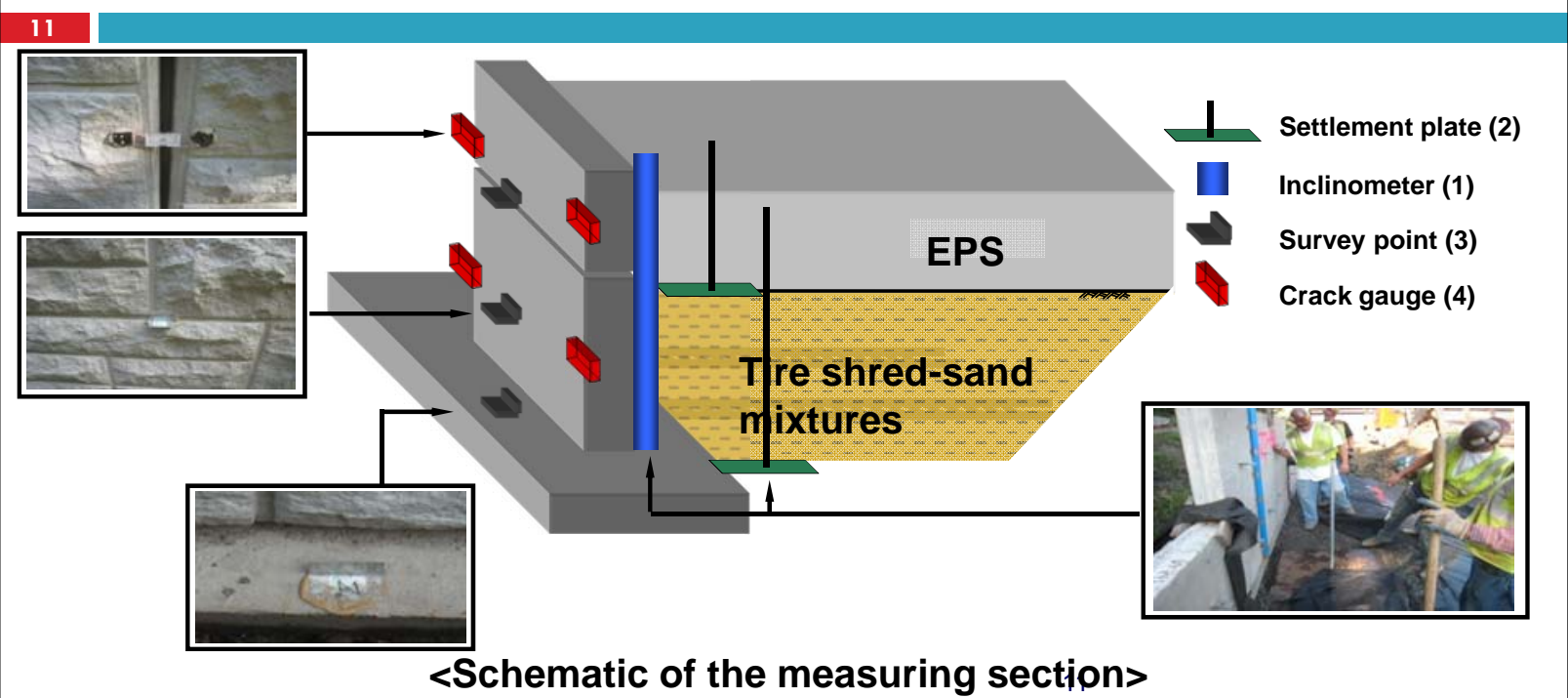

\section{Tasks - Field Monitoring}

RESULTS

- Vertical movement: Negligible movement (about $3 \mathrm{~mm}$ ) was observed after construction

- Crack gauges: Reading of less than $1 \mathrm{~mm}$ was measured

口 No significant compression of the mixture, was measured

口 No additional deformation of the wall was observed 


\section{Tasks}

LABORATORY PULLOUT TESTS

\section{Tasks - Laboratory Pullout Tests}

EQUIPMENT AT PURDUE UNIVERSITY, WEST LAFAYETTE

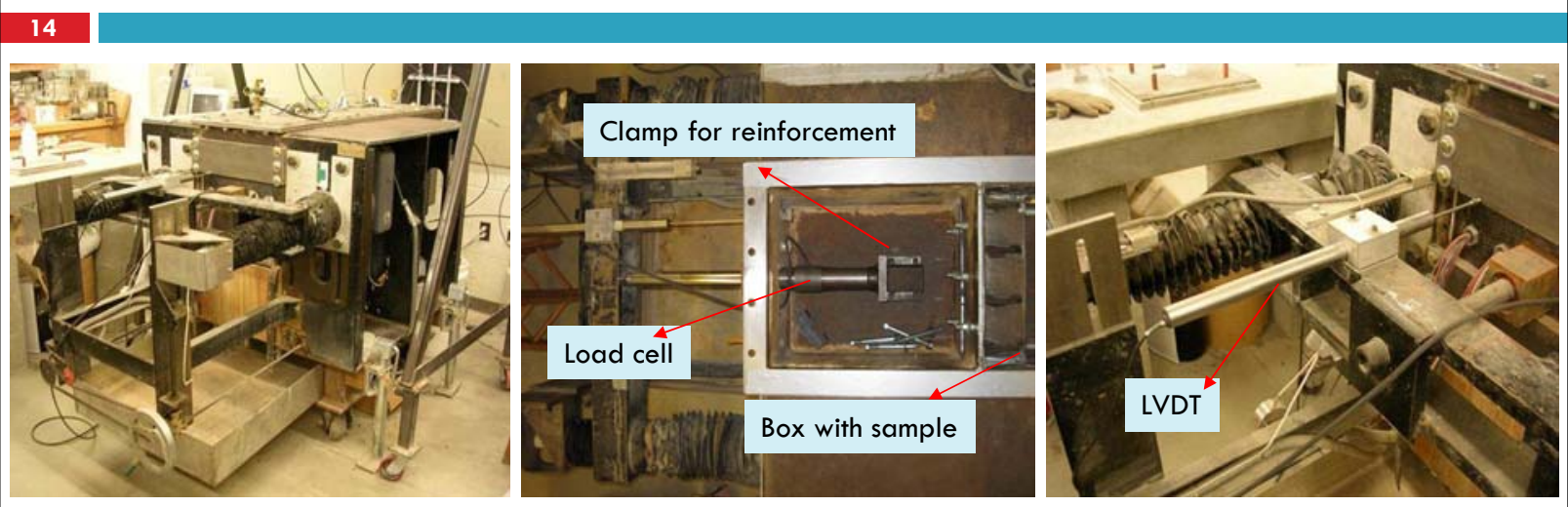

$\square$ Box dimensions: $1.0 \times 0.38 \times 0.47 \mathrm{~m}$

Shear rate: $1 \mathrm{~mm} / \mathrm{min}$ 


\section{Tasks - Laboratory Pullout Tests}

SAMPLE

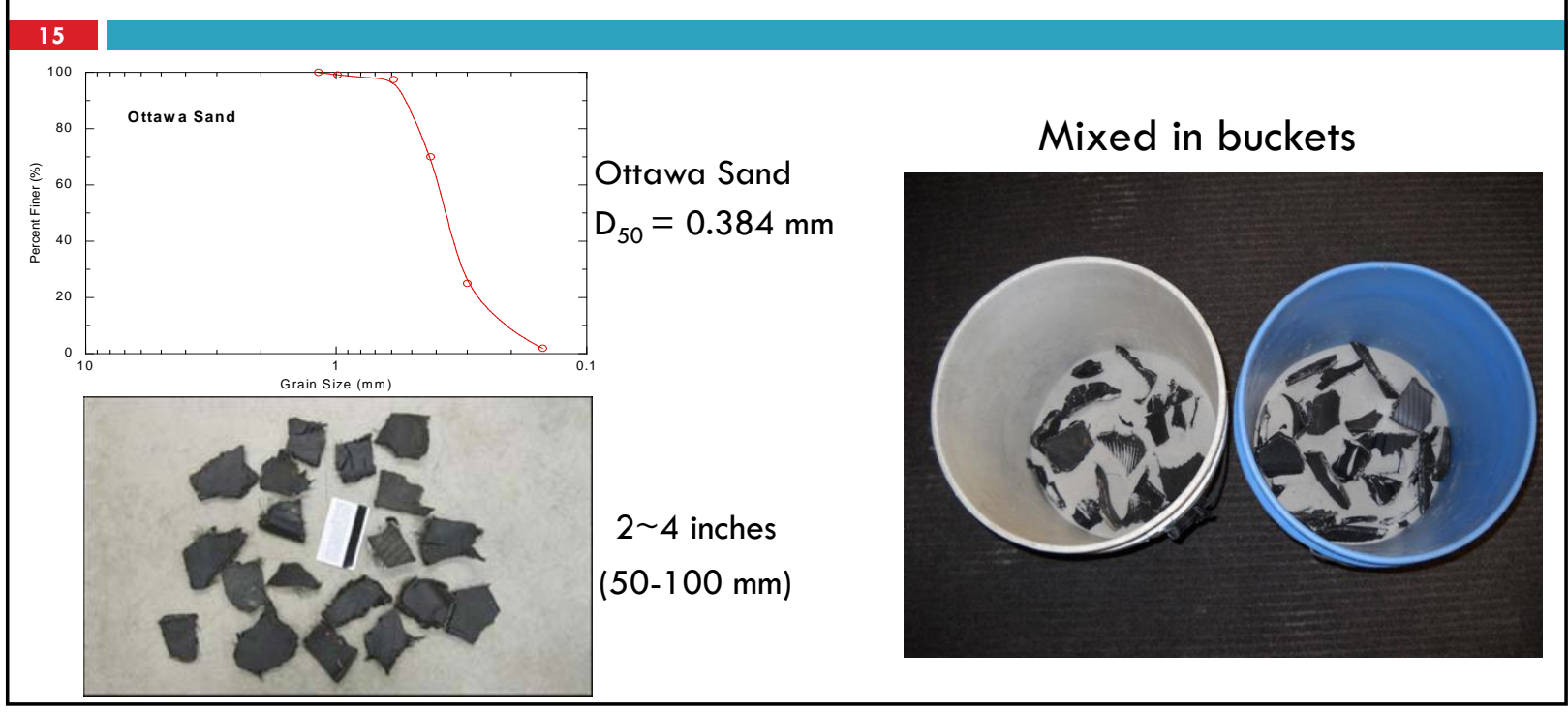

\section{Tasks - Laboratory Pullout Tests}

REINFORCEMENT

16

'Reinforcement Ladder' (Metal strap)
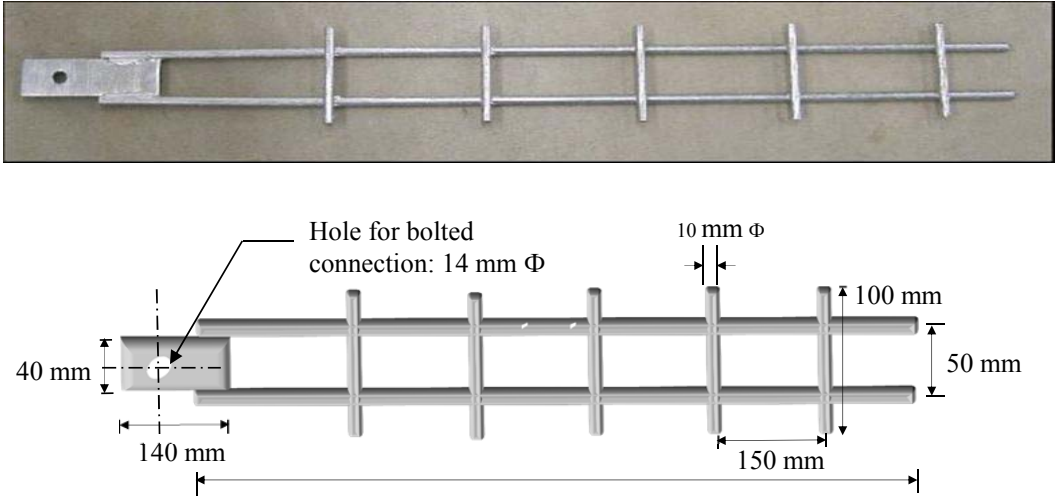

$915 \mathrm{~mm}$ 


\section{Tasks - Laboratory Pullout Tests}

\section{SCHEMATIC OF TEST}

17

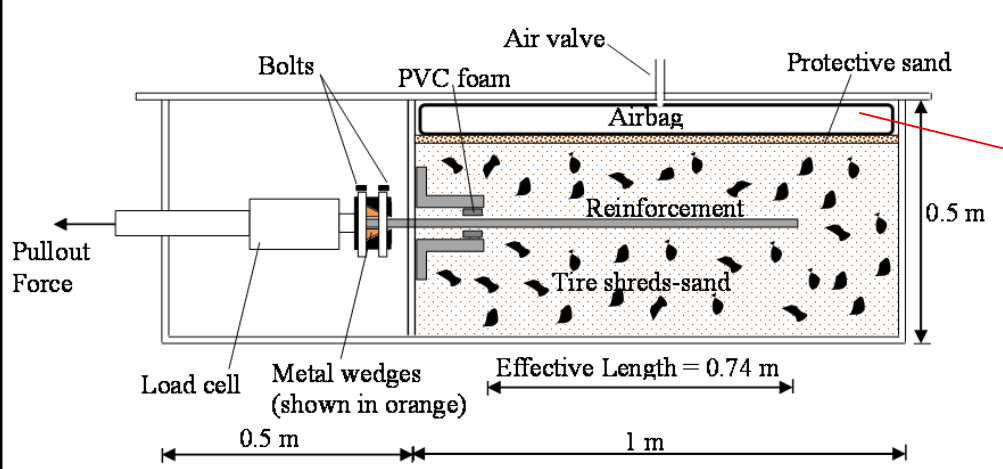

Airbag used to apply confining pressure on sample 40,65 and $90 \mathrm{kPa}$ were the pressures used

\section{Tasks - Laboratory Pullout Tests}

\section{SAMPLE PREPARATION}
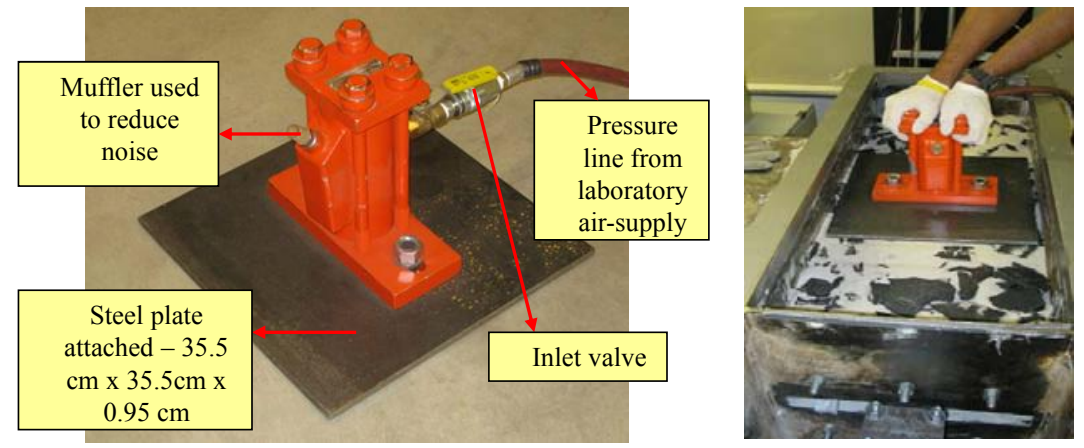

Operating pressure: $551 \mathrm{kPa}$

$\square \quad 4500$ vibrations/min

$\square \quad 4$ layers

$\square 5$ minutes/layer

Pneumatic piston-type compactor 


\section{Tasks - Laboratory Pullout Tests}

\section{TEST RESULTS - 0:1 00, 50:50 Mixing Ratios by Volume}

19
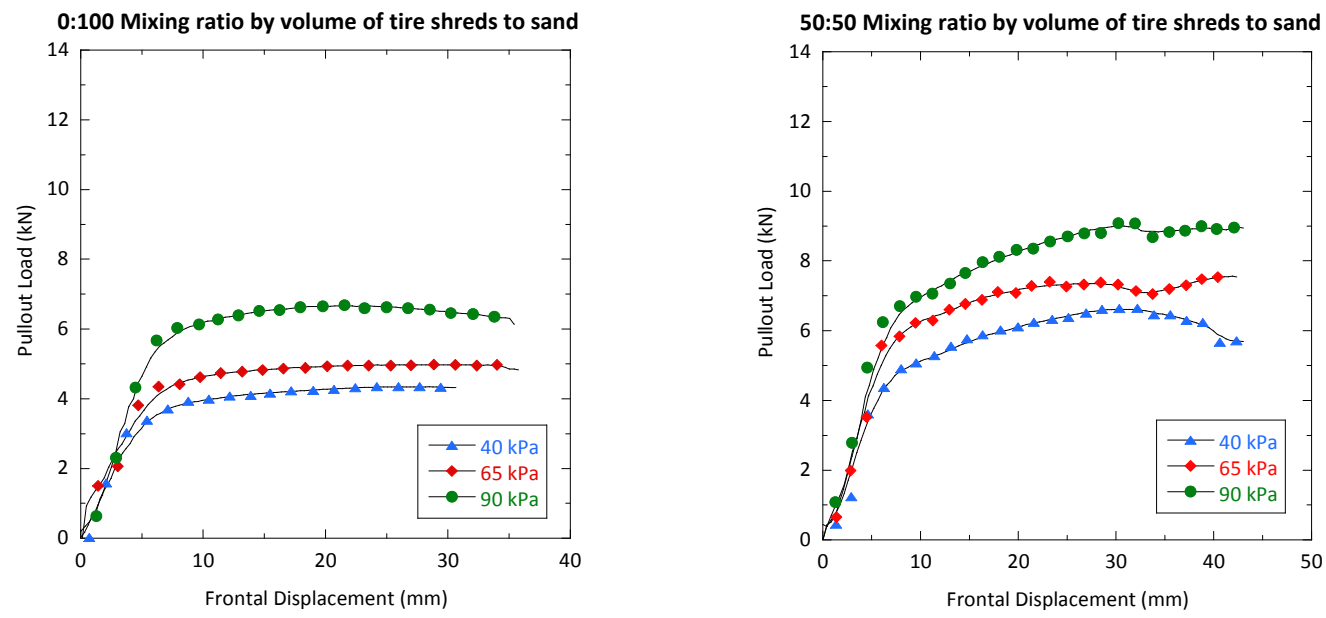

\section{Tasks - Laboratory Pullout Tests}

\section{TEST RESULTS - 60:40, 70:30 Mixing Ratios by Volume}
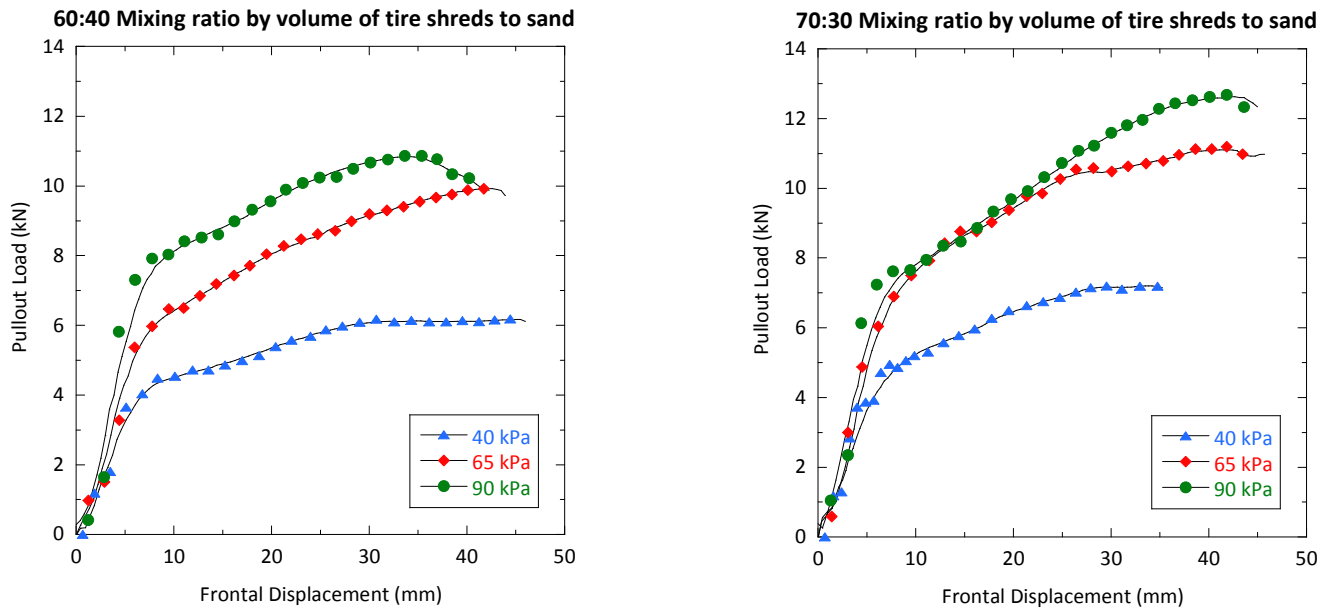


\title{
Tasks - Laboratory Pullout Tests
}

\section{TEST RESULTS - POINTS TO NOTE}

21

(a)

Pullout capacity criteria:

In case of the existence of a peak load within $20 \mathrm{~mm}$

frontal displacement, it is taken as the pullout

capacity. If there is no peak, the pullout load at 20

$\mathrm{mm}$ displacement is considered to be the pullout

capacity (FHWA 2009).
Pullout resistance factor, $F^{*}:$

$$
P_{u l t}=F^{*} \cdot \alpha \cdot \sigma_{v}^{\prime} \cdot L_{e} \cdot B \cdot C
$$

$P_{\text {ult }}=$ the pullout capacity of the reinforcement (peak load in case of existence of peak, else critical state load at test end - criteria 1)

$\mathrm{F}^{*}=$ the pullout resistance factor

$\alpha=$ correction factor to account for nonlinear shear stress distribution along the embedded length of the reinforcement (1.0 for inextensible reinforcement - Elias et. al 2001)

$\sigma_{v}^{\prime}=$ the vertical effective stress at the reinforcement-soil interface

$L_{e}=$ the embedment length of the reinforcement in the resisting zone

$B=$ the width of the metal strip (width of transverse bar $=10 \mathrm{~cm}$ )

$C=$ the effective unit perimeter of the reinforcement $(2.0$ for strip/grid type reinforcement - Elias et.al 2001)

\section{Tasks - Laboratory Pullout Tests}

\author{
TEST RESULTS - Pullout Capacity vs. Confining Pressure
}

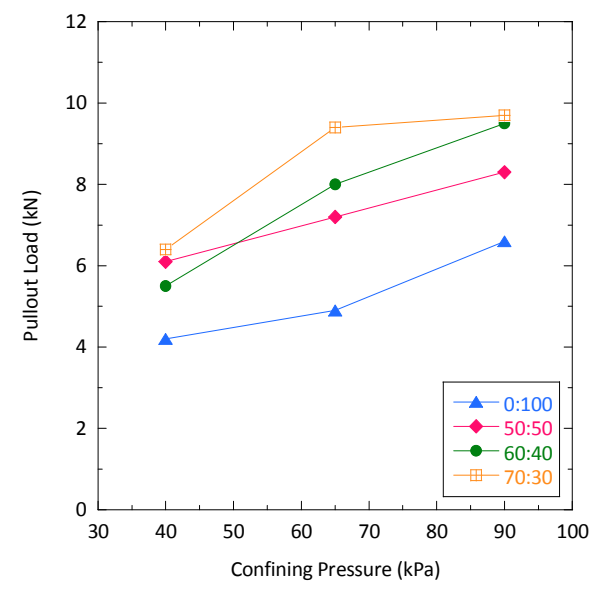




\section{Tasks - Laboratory Pullout Tests}

TEST RESULTS - Pullout Capacity vs. Tire Shred Content

23

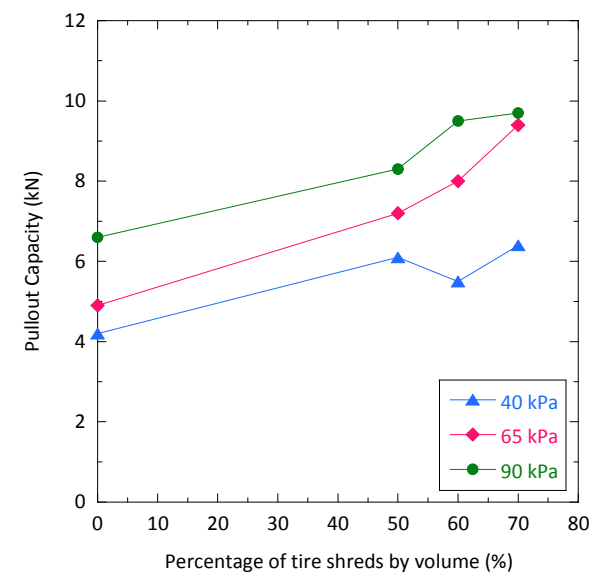

\section{Tasks - Laboratory Pullout Tests}

TEST RESULTS - Confining Pressure vs. Pullout Resistance Factor, F*

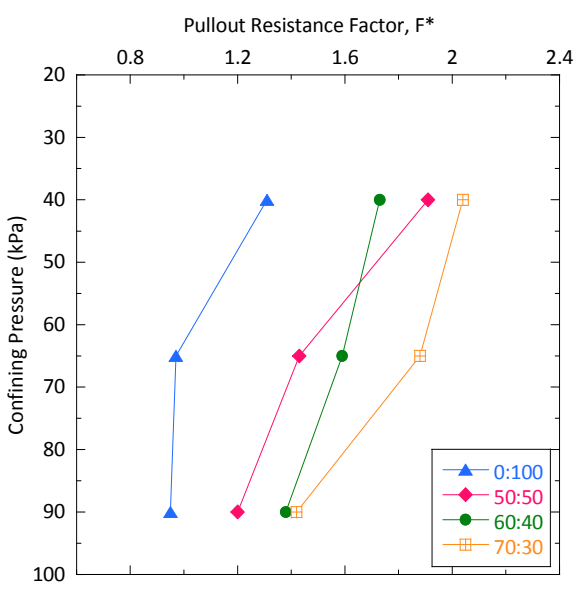




\begin{tabular}{|c|c|c|c|c|c|c|c|c|}
\hline \multicolumn{9}{|c|}{ TEST RESULTS - Summary of Pullout Test Program } \\
\hline \multicolumn{9}{|l|}{25} \\
\hline $\begin{array}{l}\text { Tire shred- } \\
\text { Sand } \\
\text { mixing ratio } \\
\quad \text { (by } \\
\text { volume) }\end{array}$ & $\begin{array}{l}\text { Tire shred- } \\
\text { Sand ratio } \\
\text { (by weight) }\end{array}$ & $\begin{array}{c}\text { Confining } \\
\text { pressure } \\
(\mathrm{kPa})\end{array}$ & $\begin{array}{c}\text { Maximum } \\
\text { dry unit } \\
\text { weight } \\
\left(\mathrm{kN} / \mathrm{m}^{3}\right) \\
(\text { Yoon 2006) }\end{array}$ & $\begin{array}{c}\text { Average dry } \\
\text { unit weight } \\
\text { in pullout } \\
\text { box }\left(\mathrm{kN} / \mathrm{m}^{3}\right)\end{array}$ & $\begin{array}{l}\text { Average } \\
\text { relative } \\
\text { compaction } \\
(\%)\end{array}$ & $\begin{array}{c}\text { Average sand- } \\
\text { matrix unit } \\
\text { weight } \\
\left(\mathrm{kN} / \mathrm{m}^{3}\right)\end{array}$ & $\begin{array}{l}\text { Reinforcement } \\
\text { ladder (metal } \\
\text { strap) pullout } \\
\text { capacity }(\mathrm{kN})\end{array}$ & $\begin{array}{l}\text { Pullout } \\
\text { resistance } \\
\text { factor, } \mathrm{F}^{*}\end{array}$ \\
\hline \multirow{3}{*}{$0: 100$} & \multirow{3}{*}{$0: 100$} & 40 & \multirow{3}{*}{18.0} & \multirow{3}{*}{17.2} & \multirow{3}{*}{95.6} & \multirow{3}{*}{17.2} & 4.2 & 1.31 \\
\hline & & 65 & & & & & 4.9 & 0.97 \\
\hline & & 90 & & & & & 6.6 & 0.95 \\
\hline \multirow{3}{*}{$50: 50$} & \multirow{3}{*}{$20: 80$} & 40 & \multirow{3}{*}{17.1} & \multirow{3}{*}{15.6} & \multirow{3}{*}{91.2} & \multirow{3}{*}{16.7} & 6.1 & 1.91 \\
\hline & & 65 & & & & & 7.2 & 1.43 \\
\hline & & 90 & & & & & 8.3 & 1.20 \\
\hline \multirow{3}{*}{$60: 40$} & \multirow{3}{*}{$25: 75$} & 40 & \multirow{3}{*}{16.6} & \multirow{3}{*}{14.8} & \multirow{3}{*}{89.1} & \multirow{3}{*}{15.8} & 5.5 & 1.73 \\
\hline & & 65 & & & & & 8.0 & 1.59 \\
\hline & & 90 & & & & & 9.5 & 1.38 \\
\hline \multirow{3}{*}{$70: 30$} & \multirow{3}{*}{$35: 65$} & 40 & \multirow{3}{*}{14.2} & \multirow{3}{*}{12.3} & \multirow{3}{*}{86.5} & \multirow{3}{*}{12.3} & 6.4 & 2.04 \\
\hline & & 65 & & & & & 9.4 & 1.88 \\
\hline & & 90 & & & & & 9.7 & 1.42 \\
\hline
\end{tabular}

\section{Tasks - Laboratory Pullout Tests}

PRACTICAL USE OF PULLOUT TEST RESULTS: 50-100 mm TS + Ottawa Sand

26

\begin{tabular}{|c|c|c|}
\hline $\begin{array}{l}\text { TS : sand mixing } \\
\text { ratio (by weight) }\end{array}$ & Equation fitted between depth $(z)$ and $F^{*}$ & $\begin{array}{l}\text { Coefficient of } \\
\text { determination, } \mathrm{R}^{2}\end{array}$ \\
\hline $20: 80$ & $\begin{array}{l}=-(z-9.9995) / 3.957 \text { for } 0 \leq z<7.7 \mathrm{~m} \\
=\tan (\phi) \text { for } z \geq 7.7 \mathrm{~m}\end{array}$ & 0.957 \\
\hline $25: 75$ & $\begin{array}{l}=-(z-17.457) / 8.512 \text { for } 0 \leq z<12.32 \mathrm{~m} \\
=\tan (\phi) \text { for } z \geq 12.32 \mathrm{~m}\end{array}$ & 0.987 \\
\hline $35: 65$ & $\begin{array}{l}=-(z-14.108) / 5.244 \text { for } 0 \leq z<12.32 \mathrm{~m} \\
=\tan (\phi) \text { for } z \geq 10.83 \mathrm{~m}\end{array}$ & 0.922 \\
\hline
\end{tabular}




\section{Tasks - Laboratory Pullout Tests}

\section{SEGREGATION OF THE MIXTURE}

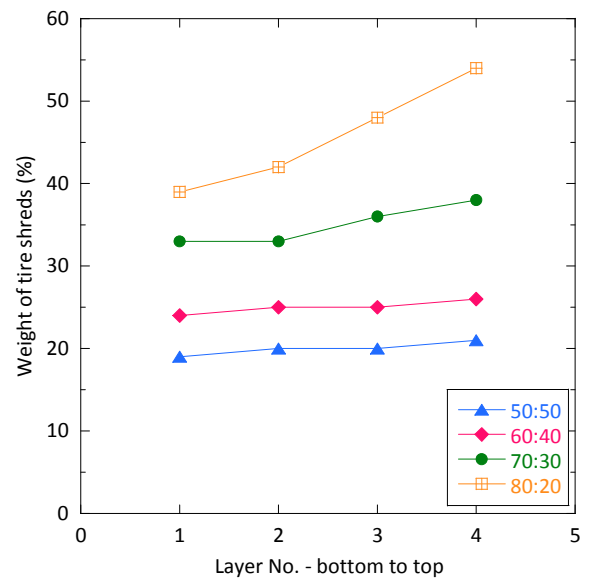

Segregation was assessed by removing the sample from the pullout box in layers and measuring the weights of TS and sand

- 50:50 and 60:40 ratios by weight of TS to sand showed practically no segregation

- 70:30 by weight of TS to sand showed very mild segregation, which could have been due to method of measurement of segregation

- 80:20 by weight of TS to sand showed considerable amount of segregation and hence results for this ratio were not presented

\section{Tasks}




\section{Tasks - Direct Shear Tests}

\section{SMALL-SCALE DIRECT SHEAR TEST (results not good)}

29

Shear machine at INDOT Research Division, West Lafayette

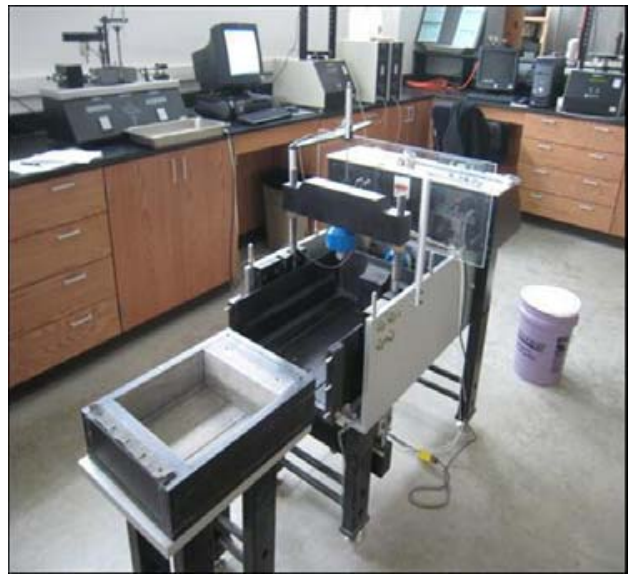

․ Dimensions: $30 \mathrm{~cm} \times 30 \mathrm{~cm} \times(10+10) \mathrm{cm}$

- Shear rate: $1 \mathrm{~mm} / \mathrm{min}$

- The small-scale tests yielded very high friction angles, in comparison to a large-scale test performed in previous research

- As a result, large-scale direct shear tests were planned

\section{Tasks - Direct Shear Tests}

LARGE-SCALE DIRECT SHEAR TEST

30

Large direct shear machine at Purdue University, West Lafayette

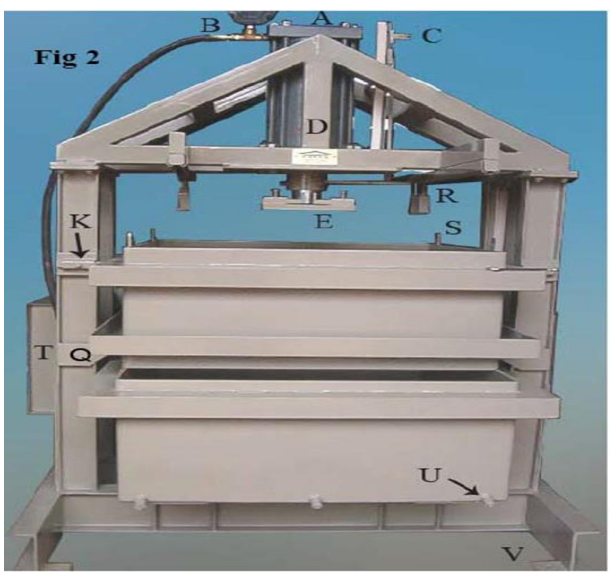

- Dimensions: Lower box $-1.2 \mathrm{~m} \times 1 \mathrm{~m} \times 0.47 \mathrm{~m}$ Upper box - $1 \mathrm{~m} \times 1 \mathrm{~m} \times 0.5 \mathrm{~m}$

口 Shear rate: $1 \mathrm{~mm} / \mathrm{min}$

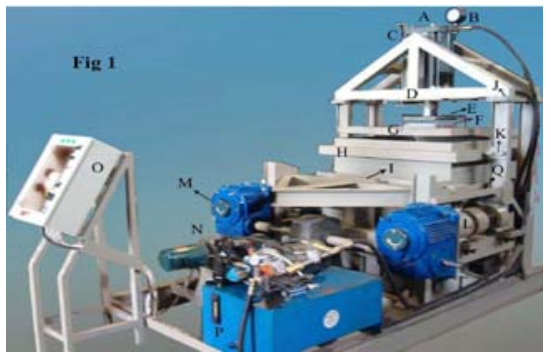




\section{Tasks - Direct Shear Tests}

\section{LARGE-SCALE DIRECT SHEAR TEST}

31
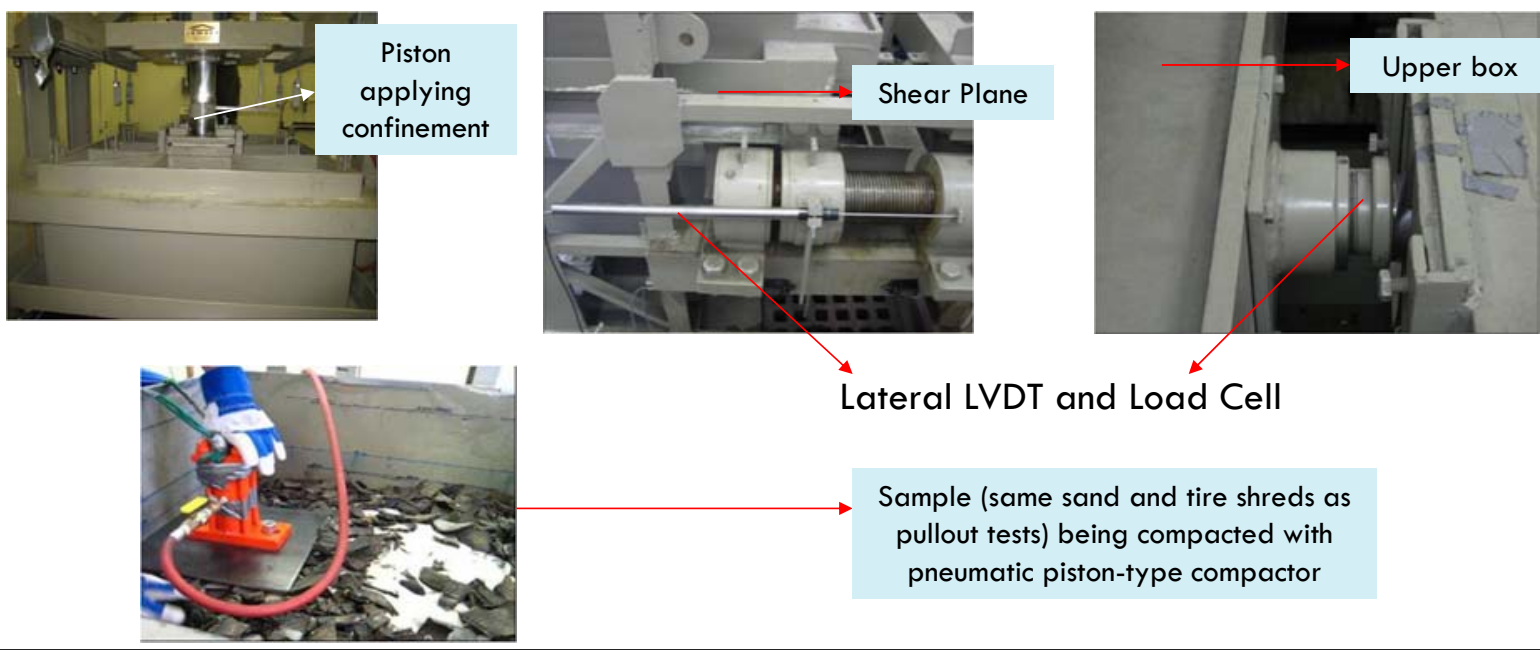

Lateral LVDT and Load Cel

Sample (same sand and tire shreds as pullout tests) being compacted with pneumatic piston-type compactor

\section{Tasks - Direct Shear Tests}

\section{LARGE-SCALE DIRECT SHEAR TEST - RESULTS}
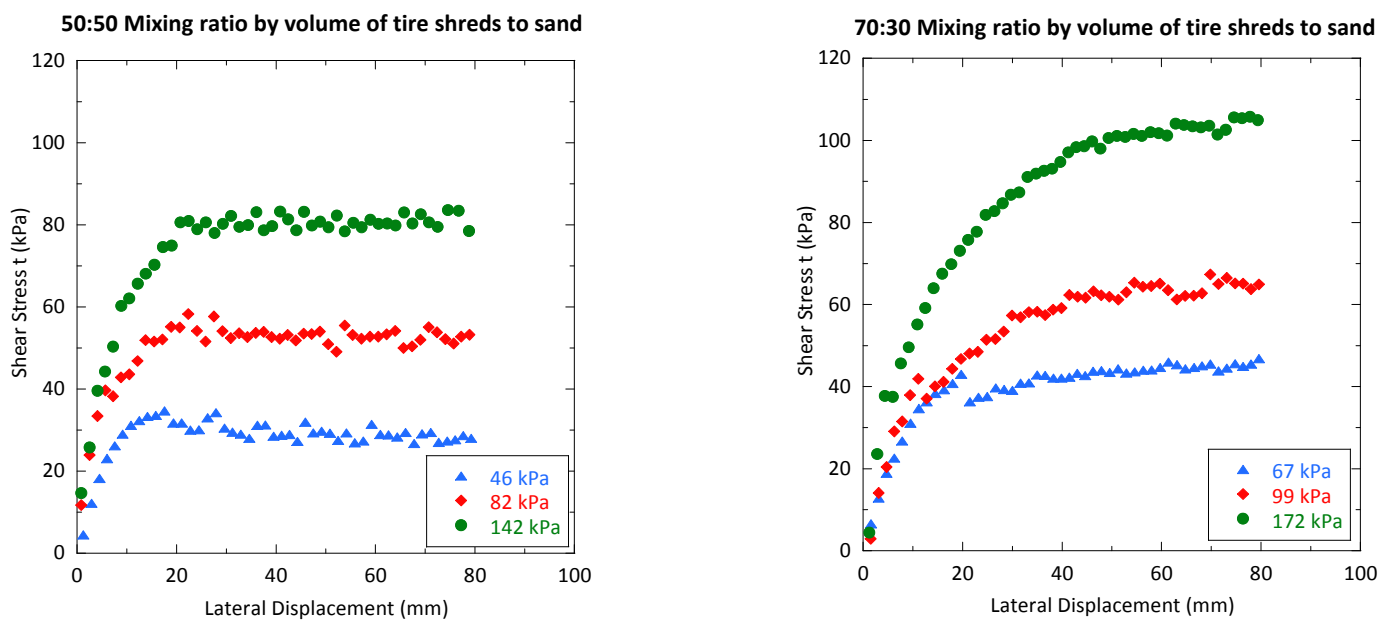


\section{Tasks - Direct Shear Tests}

LARGE-SCALE DIRECT SHEAR TEST - RESULTS

33

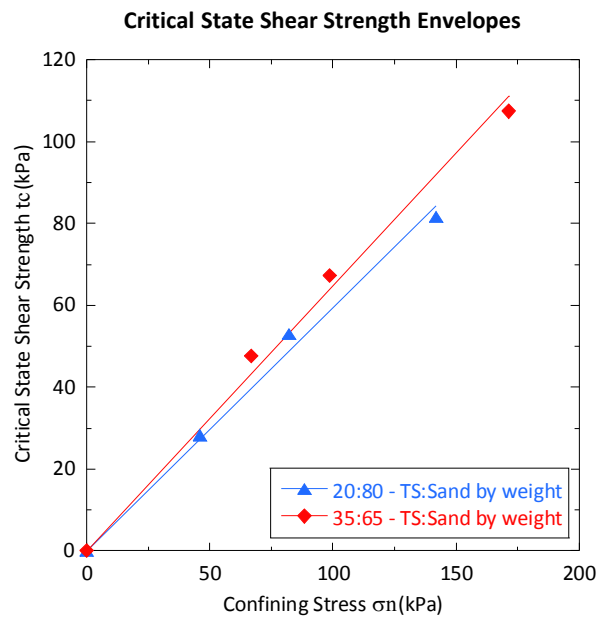

\section{Tasks - Direct Shear Tests}

LARGE-SCALE DIRECT SHEAR TEST - RESULTS

34

\begin{tabular}{|c|c|c|c|c|c|c|c|}
\hline $\begin{array}{l}\text { Size of tire } \\
\text { shreds }\end{array}$ & $\begin{array}{c}\text { Tire shred- } \\
\text { Sand ratio } \\
\text { (by volume) } \\
\sim\end{array}$ & $\begin{array}{l}\text { Tire shred- } \\
\text { Sand ratio } \\
\text { (by weight) }\end{array}$ & $\begin{array}{l}\text { Confining } \\
\text { pressure } \\
\text { range }(\mathrm{kPa})\end{array}$ & $\begin{array}{c}\text { Critical state } \\
\text { friction } \\
\text { angle, } \phi_{c}\end{array}$ & $\begin{array}{l}\text { Peak friction } \\
\text { angle, } \phi_{p}\end{array}$ & $\begin{array}{c}\text { Apparent } \\
\text { cohesion } \\
\text { intercept } \\
\text { from best } \\
\text { fit line, c } \\
(\mathrm{kPa})\end{array}$ & $\begin{array}{l}\text { Correspondi } \\
\text { ng friction } \\
\text { angle based } \\
\text { on best fit } \\
\text { line, } \phi_{c}^{\prime}\end{array}$ \\
\hline \multirow{3}{*}{$\begin{array}{c}50- \\
100 \mathrm{~mm}\end{array}$} & $50: 50$ & $25: 75$ & $46-142$ & 30.1 & 31.0 & 17.5 & 28 \\
\hline & $-60: 40$ & $20: 80$ & $60-166$ & 30.6 & 31.1 & 5.0 & 28 \\
\hline & $70: 30$ & $35: 65$ & $67-172$ & 32.0 & 32.0 & 10.3 & 30 \\
\hline
\end{tabular}




\section{Tasks}

\section{DESIGN SUMMARY}

\section{Tasks - Design Summary \\ FORMAT ADOPTED}

36

The following documents were used as a basis for determining the contents of the design summary:

\ "Plan Development Guideline for Earth Retaining Systems" -Design Services

- Section, Division of Design -Indiana Department of Highways, March 1989

"Earth Retaining Systems" : Chapter 68, from "The Indiana Design Manual 2010"

․ "Standard Specifications for Highway Bridges"-AASHTO, 17thEdition, 2002

"Geosynthetic Design and Construction Guidelines" -FHWA, April, 1998 


\section{Tasks - Design Summary}

\section{CONTENTS}

The design summary is directed towards providing the following main properties required for design of MSE walls with tire shred-sand mixtures as backfill material:

- Characteristics of tire sand and tire shreds used in preparing the mixture (Slides 37-39)

- Mixing ratio: $\quad$ 1. Conversion from weight ratio to volume mixing ratio and vice versa (Slide 40)

2. Unit weight and relative compaction of each mixing ratio (Slide 41-42)

3. Shear strength parameters for each mixing ratio (Slide 43)

- Types of reinforcement and their pullout characteristics (Slide 44-47)

\section{Tasks - Design Summary}

\section{BACKFILL REQUIREMENT - SAND}

Sand used for preparing the mixture:

- Gradation Limits:

\begin{tabular}{|c|c|}
\hline Sieve Size & Percent Passing \\
\hline 4 inches & 100 \\
\hline No. 40 & $0-60$ \\
\hline No. 20 & $0-15$ \\
\hline
\end{tabular}

- Plasticity Index: $\mathrm{PI}<6$

- The backfill material shall be free from organic and other deleterious materials, and free of shale or other soft, poor-durability particles. It shall have a Magnesium Sulfate soundness of less than 30 percent after 4 cycles 


\section{Tasks - Design Summary}

\section{BACKFILL REQUIREMENT - SAND}

39

- Electrochemical Criteria:

\begin{tabular}{|c|c|}
\hline Criteria & Test Method \\
\hline Resistivity $>300 \mathrm{ohm} \mathrm{cm}$ & AASHTO T-288 \\
\hline $\mathrm{pH}: 5-10$ & AASHTO T-289 \\
\hline Chlorides $<100 \mathrm{ppm}$ & ASTM D4327 \\
\hline Sulfates $<200 \mathrm{ppm}$ & ASTM D4327 \\
\hline Organic Content - Max. $1 \%$ & AASHTO T-267 \\
\hline
\end{tabular}

These are criteria prescribed in the literature, for choosing backfill soils of traditional types. A different set of electrochemical specifications may need to be adopted for tire shredsand mixtures.

\section{Tasks - Design Summary}

\section{BACKFILL REQUIREMENT - TIRE SHREDS}

Tire Shreds used for the mixture:

$\square$ Size of tire shreds:

ㅁ 2 4 inches $(50-100 \mathrm{~mm})$ in length in all directions

Or

․ Less than 4 inches $(100 \mathrm{~mm})$ in all directions 


\section{Tasks - Design Summary}

\section{BACKFILL REQUIREMENT - MIXTURE PROPERTIES}

41

\section{Weight to Volume Conversion}

\section{\begin{tabular}{l|l} 
Mixing Ratio by Weight & Mixing Ratio by Volume**
\end{tabular}}

$20: 80$

$25: 75$

$35: 65$

** This conversion is performed considering the minimum unit weights of Ottawa sand and 2 4 inches tire shreds used in the pullout and direct shear testing described earlier.
For example, consider a weight ratio $=20: 80$

Minimum unit weight of sand (unit weight in uncompacted state), $\gamma_{\mathrm{s}, \min }=16.6 \mathrm{kN} / \mathrm{m}^{3}$ ( 106 pcf)

$\square \quad$ Minimum unit weight of tire shreds (unit weight in uncompacted state), $\gamma_{\mathrm{TS}, \min }=4.1 \mathrm{kN} / \mathrm{m}^{3}(\sim 26 \mathrm{pcf})$

$$
\frac{\mathrm{V}_{\mathrm{TS}}}{\mathrm{V}_{\mathrm{S}}}=\frac{\frac{\mathrm{W}_{\mathrm{TS}}}{\gamma_{T S, \text { min }}}}{\frac{\mathrm{W}_{\mathrm{S}}}{\gamma_{\mathrm{S}, \text { min }}}} \longrightarrow \frac{\mathrm{V}_{\mathrm{TS}}}{\mathrm{V}_{\mathrm{Total}}}=\frac{\frac{\mathrm{W}_{\mathrm{TS}}}{\gamma_{T S, \text { min }}}}{\frac{\mathrm{W}_{\mathrm{S}}}{\gamma_{\mathrm{S}, \text { min }}}+\frac{\mathrm{W}_{\mathrm{TS}}}{\gamma_{T S, \text { min }}}}
$$

$$
\frac{\mathrm{V}_{\mathrm{TS}}}{\mathrm{V}_{\text {Total }}}=\frac{\frac{20 / 100}{4.1}}{\frac{80 / 100}{16.6}+\frac{20 / 100}{4.1}}=0.5 \longrightarrow \quad \frac{\mathrm{W}_{\mathrm{TS}}}{\mathrm{W}_{\mathrm{S}}}=\frac{20}{80} \equiv \frac{\mathrm{V}_{\mathrm{TS}}}{\mathrm{V}_{\mathrm{S}}}=\frac{50}{50}
$$

Hence, a weight ratio of $20: 80$ converts to a volume mixing ratio of $50: 50$

\section{Tasks - Design Summary}

\section{BACKFILL REQUIREMENT - MIXTURE PROPERTIES}

Mixing ratio and corresponding geotechnical properties of the mixture:

I. Unit Weight and Required RC:

* Tire shred-sand mixtures prepared with Ottawa sand $\left(D_{50}=0.384 \mathrm{~mm}\right)$ and tire shreds of size $2 \sim 4$ inches *

\begin{tabular}{|c|c|c|c|}
\hline $\begin{array}{c}\text { Mixing ratio by } \\
\text { weight }\end{array}$ & $\begin{array}{c}\text { Mixing ratio by } \\
\text { volume }\end{array}$ & $\begin{array}{c}\text { Max. dry unit } \\
\text { weight (pcf) }\end{array}$ & Required RC (\%) \\
\hline $20: 80$ & $\sim 50: 50$ & 108.8 & 95 \\
\hline $25: 75$ & $\sim 60: 40$ & 105.6 & 95 \\
\hline $35: 75$ & $\sim 70: 30$ & 90.3 & 95 \\
\hline
\end{tabular}




\section{Tasks - Design Summary}

\section{BACKFILL REQUIREMENT - MIXTURE PROPERTIES}

Mixing ratio and corresponding geotechnical properties of the mixture:

II. Unit Weight and Required RC:

* Tire shred-sand mixtures prepared with B-borrow soil $\left(D_{10}=0.24 \mathrm{~mm}, D_{30}=0.35 \mathrm{~mm}, D_{60}=0.71 \mathrm{~mm}\right)$ from SR 110 site in Marshall County, and tire shreds of 2 inches nominal size *

\begin{tabular}{|c|c|c|c|}
\hline $\begin{array}{c}\text { Mixing ratio by } \\
\text { weight }\end{array}$ & $\begin{array}{c}\text { Mixing ratio by } \\
\text { volume }^{+}\end{array}$ & $\begin{array}{l}\text { Max. dry unit } \\
\text { weight (pcf)* }\end{array}$ & Required RC (\%) \\
\hline $25: 75$ & $\sim 50: 50$ & 103 & 95 \\
\hline $35: 65$ & $\sim 60: 40$ & 96 & 95 \\
\hline $45: 65$ & $\sim 70: 30$ & 90 & 95 \\
\hline
\end{tabular}

\section{Tasks - Design Summary}

\section{BACKFILL REQUIREMENT - MIXTURE PROPERTIES}

Mixing ratio and corresponding geotechnical properties of the mixture:

* Tire shred-sand mixtures prepared with Ottawa sand $\left(D_{50}=0.384 \mathrm{~mm}\right)$ and tire shreds of size $2 \sim 4$ inches * Shear Strength

\begin{tabular}{|c|c|c|c|c|c|}
\hline $\begin{array}{c}\text { Mixing ratio } \\
\text { by weight }\end{array}$ & $\begin{array}{c}\text { Mixing ratio } \\
\text { by volume }\end{array}$ & $\phi_{\mathrm{c}}$ (degrees) & $\phi_{\mathrm{p}}$ (degrees) & $\begin{array}{c}\text { Cohesive } \\
\text { intercept (from } \\
\text { best fit line), c } \\
\text { (kPa) }\end{array}$ & $\begin{array}{c}\text { Corresponding } \\
\phi \text { from best fit } \\
\text { line }\end{array}$ \\
\hline $20: 80$ & $\sim 50: 50$ & 30.1 & 31.0 & 14.5 & 27.1 \\
\hline $25: 75$ & $\sim 60: 40$ & 30.6 & 31.1 & 17.5 & 28.0 \\
\hline $35: 75$ & $\sim 70: 30$ & 32.0 & 32.0 & 10.3 & 29.6 \\
\hline
\end{tabular}




\section{Tasks - Design Summary}

REINFORCEMENTS - TYPE AND PULLOUT CHARACTERISTICS

45

\section{REINFORCEMENT LADDERS (METAL STRAPS)}

Tire shred-

Sand Tire shred-

mixing ratio Sand ratio

(by (by weight)

volume)

$\quad(\mathrm{kPa})$

Confining

Maximum

dry unit

weight

$\left(\mathrm{kN} / \mathrm{m}^{3}\right)$

(Yoon 2006)

Average dry unit weight

in pullout

box $\left(\mathrm{kN} / \mathrm{m}^{3}\right)$

\section{Average}

relative

compaction

$(\%)$
Average sandmatrix unit

weight

$\left(\mathrm{kN} / \mathrm{m}^{3}\right)$
Reinforcemen

ladder (metal

strap) pullout capacity $(\mathrm{kN})$
Pullout resistance factor, $\mathrm{F}^{*}$

\begin{tabular}{|c|c|c|c|c|c|c|c|c|}
\hline $0: 100$ & $0: 100$ & $\begin{array}{l}40 \\
65 \\
90 \\
\end{array}$ & 18.0 & 17.2 & 95.6 & 17.2 & $\begin{array}{l}4.2 \\
4.9 \\
6.6 \\
\end{array}$ & $\begin{array}{l}.31 \\
0.97 \\
0.95 \\
\end{array}$ \\
\hline $50: 50$ & $20: 80$ & $\begin{array}{l}40 \\
65 \\
90 \\
\end{array}$ & 17.1 & 15.6 & 91.2 & 16.7 & $\begin{array}{l}6.1 \\
7.2 \\
8.3 \\
\end{array}$ & $\begin{array}{l}1.91 \\
1.43 \\
1.20 \\
\end{array}$ \\
\hline $60: 40$ & $25: 75$ & $\begin{array}{l}40 \\
65 \\
90 \\
\end{array}$ & 16.6 & 14.8 & 89.1 & 15.8 & $\begin{array}{l}5.5 \\
8.0 \\
9.5 \\
\end{array}$ & $\begin{array}{l}1.73 \\
1.59 \\
1.38 \\
\end{array}$ \\
\hline $70: 30$ & $35: 65$ & $\begin{array}{l}40 \\
65 \\
90\end{array}$ & 14.2 & 12.3 & 86.5 & 12.3 & $\begin{array}{l}6.4 \\
9.4 \\
9.7\end{array}$ & $\begin{array}{l}2.04 \\
1.88 \\
1.42\end{array}$ \\
\hline
\end{tabular}

\section{Tasks - Design Summary}

REINFORCEMENTS - TYPE AND PULLOUT CHARACTERISTICS - RIBBED METAL STRIP

46

\begin{tabular}{|c|c|c|c|c|c|c|c|c|}
\hline $\begin{array}{c}\text { Size of tire } \\
\text { pieces }\end{array}$ & $\begin{array}{c}\text { Tire } \\
\text { shred- } \\
\text { sand } \\
\text { ratio (by } \\
\text { weight) }\end{array}$ & $\begin{array}{c}\text { Tire } \\
\text { shred- } \\
\text { sand ratio } \\
\text { (by } \\
\text { volume) }\end{array}$ & $\begin{array}{c}\text { Confining } \\
\text { pressure } \\
(\mathrm{kPa})\end{array}$ & $\begin{array}{c}\text { Maximum } \\
\text { dry unit } \\
\text { weight } \\
\left(\mathrm{kN} / \mathrm{m}^{3}\right) \\
(\text { Yoon } \\
2006) \\
\end{array}$ & $\begin{array}{l}\text { Average } \\
\text { dry unit } \\
\text { weight in } \\
\text { pullout } \\
\text { box }\end{array}$ & $\begin{array}{c}\text { Relative } \\
\text { compaction } \\
(\%)\end{array}$ & $\begin{array}{l}\text { Ribbed metal } \\
\text { strip pullout } \\
\text { capacity }(\mathrm{kN})\end{array}$ & $\begin{array}{c}\text { Pullout } \\
\text { resistance } \\
\text { factor, } \\
\mathrm{F}^{*}\end{array}$ \\
\hline $\begin{array}{c}\text { Ottawa } \\
\text { Sand }\end{array}$ & $0: 100$ & $0: 100$ & $\begin{array}{l}40 \\
65 \\
90\end{array}$ & 18.0 & 17.2 & 95.5 & $\begin{array}{l}4.4 \\
5.2 \\
5.8\end{array}$ & $\begin{array}{l}1.49 \\
1.08 \\
0.87\end{array}$ \\
\hline $\begin{array}{l}9.5 \mathrm{~mm} \\
\text { size chips }\end{array}$ & $25: 75$ & $50: 50$ & $\begin{array}{l}40 \\
65 \\
90 \\
\end{array}$ & 15.2 & 14.3 & 94.3 & $\begin{array}{l}3.2 \\
4.0 \\
4.9 \\
\end{array}$ & $\begin{array}{l}1.08 \\
0.83 \\
0.74 \\
\end{array}$ \\
\hline $50-100 \mathrm{~mm}$ & $25: 75$ & $58: 42$ & $\begin{array}{l}40 \\
65 \\
90 \\
\end{array}$ & 16.6 & 14.7 & 88.5 & $\begin{array}{l}3.1 \\
3.7 \\
4.4 \\
\end{array}$ & $\begin{array}{l}1.05 \\
0.77 \\
0.66 \\
\end{array}$ \\
\hline $\begin{array}{c}100- \\
200 \mathrm{~mm}\end{array}$ & $25: 75$ & $69: 31$ & $\begin{array}{l}40 \\
65 \\
90 \\
\end{array}$ & 16.5 & 14.2 & 86.2 & $\begin{array}{l}3.0 \\
3.4 \\
3.8 \\
\end{array}$ & $\begin{array}{l}1.01 \\
0.71 \\
0.57 \\
\end{array}$ \\
\hline
\end{tabular}




\section{Tasks - Design Summary}

REINFORCEMENTS - TYPE AND PULLOUT CHARACTERISTICS

\begin{tabular}{ccc}
\hline GEOGRIDS & \\
\hline Property & Unit & Machine Direction (MD) Value \\
\hline True initial modulus & $\mathrm{kN} / \mathrm{m}$ & 1580 \\
\hline Tensile strength at 5\% strain & $\mathrm{kN} / \mathrm{m}$ & 52 \\
\hline Long-term allowable load in sand silt and clay & $\mathrm{kN} / \mathrm{m}$ & 45.2 \\
\hline Long-term allowable load in well-graded sand & $\mathrm{kN} / \mathrm{m}$ & 43.9 \\
\hline Long-term allowable load in aggregate & $\mathrm{kN} / \mathrm{m}$ & 43.1 \\
\hline Junction strength & $\mathrm{kN} / \mathrm{m}$ & 105 \\
\hline
\end{tabular}

\begin{tabular}{|c|c|c|c|c|c|c|c|c|}
\hline \multicolumn{9}{|c|}{ REINFORCEMENTS - TYPE AND PULLOUT CHARACTERISTICS - GEOGRIDS } \\
\hline 48 & & & & & & & & \\
\hline & $\begin{array}{l}\text { Size of } \\
\text { tire } \\
\text { pieces }\end{array}$ & $\begin{array}{l}\text { Tire shred- } \\
\text { Sand ratio } \\
\text { (by weight) }\end{array}$ & $\begin{array}{c}\text { Confining } \\
\text { Pressure } \\
(\mathrm{kPa})\end{array}$ & $\begin{array}{c}\text { Maximum Dry } \\
\text { Unit Weight } \\
\left(\mathrm{kN} / \mathrm{m}^{3}\right) \\
(\text { Yoon 2006) }\end{array}$ & $\begin{array}{c}\text { Average dry } \\
\text { unit weight } \\
\text { in pullout } \\
\text { box }\end{array}$ & $\begin{array}{c}\text { Relative } \\
\text { compaction } \\
(\%)\end{array}$ & $\begin{array}{c}\text { Average } \\
\text { Sand-matrix } \\
\text { unit weight }\end{array}$ & $\begin{array}{c}\text { Geogrid } \\
\text { pullout } \\
\text { capacity } \\
(\mathrm{kN} / \mathrm{m}) \\
\end{array}$ \\
\hline & $\begin{array}{l}\text { Ottawa } \\
\text { Sand }\end{array}$ & $25: 75$ & $\begin{array}{c}40 \\
70 \\
100\end{array}$ & 18.0 & 17.4 & 96.7 & 17.4 & $\begin{array}{c}23.8 \\
32.6 \\
42\end{array}$ \\
\hline & $\begin{array}{l}9.5 \mathrm{~mm} \\
\text { size } \\
\text { chips }\end{array}$ & $25: 75$ & $\begin{array}{c}40 \\
70 \\
100\end{array}$ & 15.2 & 14.5 & 95.4 & 16.9 & $\begin{array}{l}23.6 \\
34.5 \\
40.1\end{array}$ \\
\hline & $\begin{array}{l}50-100 \\
\text { mm } \\
\text { shreds }\end{array}$ & $25: 75$ & $\begin{array}{c}40 \\
70 \\
100\end{array}$ & 16.6 & 15.1 & 90.8 & 16.4 & $\begin{array}{l}19.7 \\
25.6 \\
30.7\end{array}$ \\
\hline & $\begin{array}{c}100-200 \\
\text { mm } \\
\text { shreds }\end{array}$ & $25: 75$ & $\begin{array}{c}40 \\
70 \\
100\end{array}$ & 16.5 & 14.6 & 88.5 & 16.0 & $\begin{array}{l}15.8 \\
22.0 \\
32.1\end{array}$ \\
\hline
\end{tabular}


THANK YOU 


\section{DESIGN SUMMARY FOR THE PRACTICAL USE OF TIRE SHRED-SAND MIXTURES}

\subsection{Introduction}

Various design parameters are required for the practical use of tire shred-sand mixtures. This document provides the essential properties of tire shred-sand mixtures, which can be used as criteria for design when the mixture is used as backfill material for mechanically stabilized earth (MSE) walls. In order to prepare this design summary existing design manuals for MSE walls were used as reference for certain applicable criteria while other properties were determined from laboratory tests. The design summary provides the following:

1. Size characteristics and index properties of tire shreds and sand used to prepare the mixture

2. Mixing Ratio:

(i) Conversion from weight ratio to volume mixing ratio and vice-versa

(ii) Unit weight and relative compaction requirements at each mixing ratio

(iii) Shear strength parameters at each mixing ratio

3. Pullout characteristics for different types of reinforcements embedded in tire shredsand mixtures. 


\subsection{Size and, Index and Chemical properties of Tire Shred-Sand Backfill}

\subsubsection{Sand}

\section{Gradation Limits:}

The sand used for preparing the mixture should qualify the size criteria prescribed in Table 1.1.

Table 1.1 Gradation limits of the sand to be used in preparing tire shred-sand mixtures (FHWA 2009)

\begin{tabular}{|c|c|}
\hline Sieve Size & Percent Passing \\
\hline 4 inches & 100 \\
\hline No. 40 & $0-60$ \\
\hline No. 20 & $0-15$ \\
\hline
\end{tabular}

Plasticity Index:

The plasticity index of the sand should not exceed 6 (FHWA 2009).

Apart from the gradation and plasticity index requirements, the sand used in preparing the mixture for backfill should be free of organic and other deleterious materials. The sand should not contain shale or other soft and low-durability particles. The sand should also have a Magnesium Sulfate soundness of less than 30 percent after 4 cycles (FHWA 2009). 


\section{Electrochemical Criteria:}

The electrochemical criteria for the sand is shown in Table 1.2. These criteria are based on existing recommendations for backfill material for MSE walls. However, tests may need to be conducted to determine the electrochemical requirements for tire shred-sand mixtures.

Table 1.2 Electrochemical criteria for sand (FHWA 2009)

\begin{tabular}{|c|c|}
\hline Criteria & Test Method \\
\hline Resistivity $>300 \mathrm{ohm} \mathrm{cm}$ & AASHTO T-288 \\
\hline $\mathrm{pH}: 5-10$ & AASHTO T-289 \\
\hline Chlorides $<100 \mathrm{ppm}$ & ASTM D4327 \\
\hline Sulfates $<200 \mathrm{ppm}$ & ASTM D4327 \\
\hline Organic Content - Max. $1 \%$ & AASHTO T-267 \\
\hline
\end{tabular}

\subsubsection{Tire Shreds}

The tire shreds used for preparing the mixture are of size 2 to 4 inches (50 to $100 \mathrm{~mm}$ ), measured along the longest length between any two points on a given tire shred. The properties of tire shred-sand mixtures presented in this summary correspond to mixtures prepared with tire shreds of size 2 to 4 inches. Other lengths of tire shreds 
may be used if properties have been determined for mixtures prepared using the corresponding size of tire shreds.

\subsection{Properties of Tire Shred-Sand Mixtures}

\subsubsection{Weight to Volume Conversion (vice-versa)}

The weight ratios can be converted to their corresponding volume mixing ratios using the minimum dry unit weight of pure tire shreds and sand (Balunaini 2009). The minimum unit weight of sand or tire shreds refers to the unit weight of sand or tire shreds measured in an uncompacted state within a compaction mold. Here, it is assumed that the minimum unit weight of pure sand closely resembles the individual unit weight of sand within the mixture. Similarly, it is assumed that the minimum unit weight of tire shreds closely resembles the individual unit weight of tire shreds within the mixture. We will now look at an example for this weight ratio to volume mixing ratio conversion for the tire shred-sand mixture.

Example 1.1:

Consider a weight ratio $=W_{T S}: W_{S}=20: 80$ by weight of tire shreds to sand. $W_{\text {Total }}$ is the total weight of the sample. Let $V_{T S}: V_{S}$ be the ratio by volume of tire shreds to sand. $V_{\text {Total }}$ is the total volume occupied by the sample.

From Table 3.1 we know that,

Minimum unit weight of sand (unit weight in uncompacted state), $\gamma_{S, \min }=16.6 \mathrm{kN} / \mathrm{m}^{3}$ 
Minimum unit weight of tire shreds (unit weight in uncompacted state), $\gamma_{T S \text {, min }}=$

\section{$4.1 \mathrm{kN} / \mathrm{m}^{3}$}

Now, from the definition of unit weight,

$$
V=\frac{W}{\gamma}
$$

The weight ratio considered is 20:80, by weight of tire shreds to sand,

$$
\frac{W_{T S}}{W_{S}}=\frac{20}{80}
$$

Combining the above two equations and from the assumption that the minimum unit weights of individual materials are close to the unit weights of individual materials in the mixture,

$$
\frac{V_{T S}}{V_{S}}=\frac{\frac{W_{T S}}{\gamma_{T S, \min }}}{\frac{W_{S}}{\gamma_{S, \text { min }}}}
$$

$$
\text { and also, } V_{\text {Total }}=V_{T S}+V_{S}
$$

Combining the above two equations

$$
\frac{V_{T S}}{V_{\text {Total }}}=\frac{\frac{W_{T S}}{\gamma_{T S, \min }}}{\frac{W_{T S}}{\gamma_{T S, \min }}+\frac{W_{S}}{\gamma_{S, \text { min }}}}
$$

Substituting the values for all the quantities on the right hand side of the equation,

$$
\frac{V_{T S}}{V_{\text {Total }}}=\frac{\frac{20 / 100}{4.1}}{\frac{20 / 100}{4.1}+\frac{80 / 100}{16.6}}=0.5
$$




$$
\text { and also, } V_{\text {Total }}=V_{T S}+V_{S}
$$

As a result,

$$
\frac{V_{T S}}{V_{S}}=\frac{50}{50}
$$

Therefore,

$$
\frac{W_{T S}}{W_{S}}=\frac{20}{80} \equiv \frac{V_{T S}}{V_{S}}=\frac{50}{50}
$$

Hence, a weight ratio of 20:80 converts to a volume ratio of 50:50, tire shreds to sand.

Table shows the conversion of the tire shred-to-sand weight ratios of the mixtures considered in this study, to the corresponding volume mixing ratios

Table 1.3 Conversion from weight ratios to volume ratios calculated using the minimum unit weight of tire shreds and sand

\begin{tabular}{cc}
\hline $\begin{array}{c}\text { Weight ratio } \\
\text { (TS:Sand) }\end{array}$ & $\begin{array}{c}\text { Volume ratio (TS:Sand) } \\
\text { (Calculated using } \gamma_{S, \min } \text { and } \gamma_{T S, \min } \text { ) }\end{array}$ \\
\hline $20: 80$ & $50: 50$ \\
$25: 75$ & $58: 42$ \\
$35: 65$ & $69: 31$ \\
$45: 55$ & $77: 23$ \\
\hline
\end{tabular}

Hence $20: 80,25: 75$ and $35: 65$ mixing ratios by weight approximately convert to volume mixing ratios of 50:50, 60:40 and 70:30, tire shreds to sand. 


\subsubsection{Dry Unit Weight}

The dry unit weights of tire shred-sand mixtures, prepared with tire shreds of size 50$100 \mathrm{~mm}$ ( 2 to 4 inches $)$ and Ottawa sand $\left(\mathrm{D}_{50}=0.384 \mathrm{~mm}\right)$ at different mixing ratios by weight, are provided in Figure 1.1. Yoon (2006) proposed an optimum mixing ratio of $25: 75$ by weight of tire shreds to sand ( $\sim 60: 40$ by volume mixing ratio of tire shreds to sand) that corresponds to the least void ratio. Table 1.4 provides the dry unit weights at the optimum mixing ratio (in terms of volume mixing ratio) and at adjacent mixing ratios, which are most likely to be used in practice.

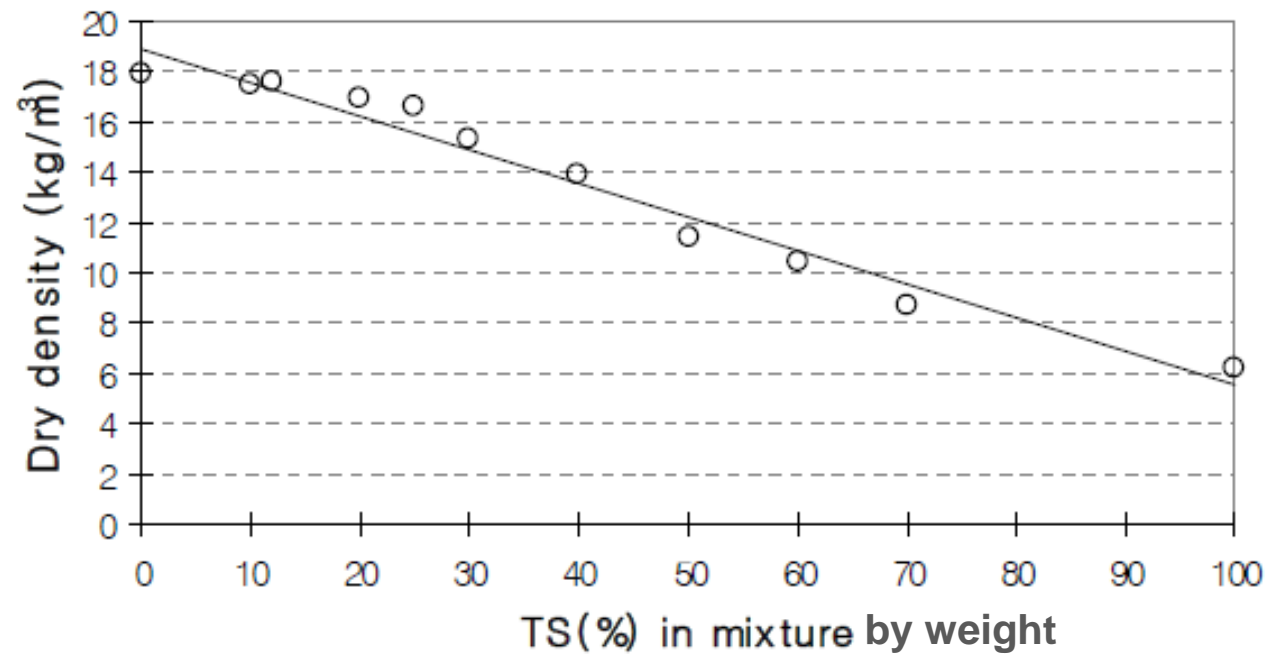

Figure 1.1 Variation of dry unit weight with percentage of tire shreds in the mixture (by weight) for tire shred sand mixtures prepared with 2 to 4 inches tire shreds and Ottawa sand (Yoon 2006) 
Table 1.4 Unit weights of tire shred-sand mixtures (2-4 in shreds + Ottawa sand) at mixing ratios likely to be used in practice

\begin{tabular}{|c|c|c|}
\hline $\begin{array}{c}\text { Mixing ratio by } \\
\text { weight }\end{array}$ & $\begin{array}{c}\text { Volume mixing } \\
\text { ratio }\end{array}$ & $\begin{array}{c}\text { Max. dry unit } \\
\text { weight (pcf) }\end{array}$ \\
\hline $20: 80$ & $\sim 50: 50$ & 108.8 \\
\hline $25: 75$ & $\sim 60: 40$ & 105.6 \\
\hline $35: 75$ & $\sim 70: 30$ & 90.3 \\
\hline
\end{tabular}

Now, we will look at the unit weights of tire shred-sand mixtures prepared with $50 \mathrm{~mm}$ (2 inch) nominal size tire shreds and B-borrow sand (at 4\% moisture content) obtained from SR-110 site in Marshall County, Indiana. Figure 1.2 shows the variation of unit weight with tire shred content in the mixture. The below equation can be used to calculate the unit weight of the mixture at different tire shred contents by weight:

$$
\begin{aligned}
& \gamma=17.875-0.0549(\mathrm{TS})-0.000629(\mathrm{TS})^{2} \\
& \rho=113.748-0.3492(\mathrm{TS})-0.00400(\mathrm{TS})^{2}
\end{aligned}
$$

Where, TS is the percentage of tire shreds in the mixture by weight

Table 1.5 provides the unit weights at the optimum mixing ratio (in terms of volume mixing ratio) and at adjacent mixing ratios, which are most likely to be used in practice. 


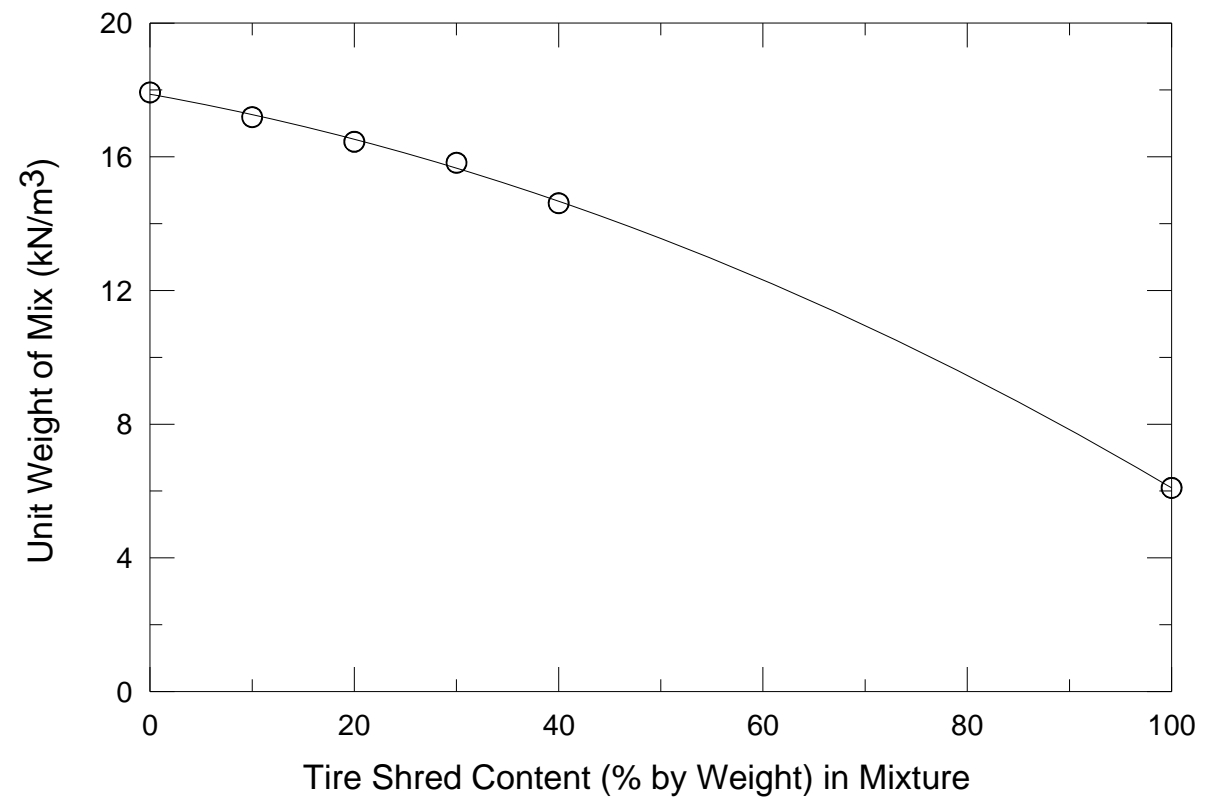

Figure 1.2 Variation of dry unit weight with percentage of tire shreds in the mixture (by weight) for tire shred sand mixtures prepared with 2 inch tire shreds and B-borrow sand

Table 1.5 Unit weights of tire shred-sand mixtures ( 2 in shreds + B-borrow sand) at mixing ratios likely to be used in practice

\begin{tabular}{|c|c|c|}
\hline $\begin{array}{c}\text { Mixing ratio by } \\
\text { weight }\end{array}$ & $\begin{array}{c}\text { Volume mixing } \\
\text { ratio }\end{array}$ & $\begin{array}{c}\text { Max. dry unit } \\
\text { weight (pcf) }\end{array}$ \\
\hline $25: 75$ & $\sim 50: 50$ & 103 \\
\hline $35: 65$ & $\sim 60: 40$ & 96 \\
\hline $45: 65$ & $\sim 70: 30$ & 90 \\
\hline
\end{tabular}

It should be noted that the conversions from weight ratio to volume mixing ratio are different in Table 1.4 and 1.5 because of the different size of tire shreds and the different sand used. In Table 1.4 the minimum unit weights of tire shreds and sand are 
as assumed in Example 1.1 of Section 1.3.1, while in Table 1.5 the minimum unit weights of tire shreds and sand are assumed to be $31 \mathrm{pcf}$ and $90 \mathrm{pcf}$ respectively. The minimum unit weights are used to perform the weight to volume mixing ratio conversion as shown in Example 1.1.

\subsubsection{Shear Strength}

The shear strength parameters for mixing ratios that are likely to be used in practice are provided in Table 1.6. These parameters were determined through large scale direct shear tests. The dimensions of the upper box are $1.2 \mathrm{~m} \times 1.0 \mathrm{~m} \times 0.47 \mathrm{~m}$ while the dimensions of the lower box are $1.0 \mathrm{~m} \times 1.0 \mathrm{~m} \times 0.5 \mathrm{~m}$. The shear rate was $1 \mathrm{~mm} / \mathrm{min}$.

Table 1.6 Shear Strength parameters for tire shred-sand mixtures

\begin{tabular}{|c|c|c|c|c|c|}
\hline $\begin{array}{c}\text { Mixing } \\
\text { ratio by } \\
\text { weight }\end{array}$ & $\begin{array}{c}\text { Mixing } \\
\text { ratio by } \\
\text { volume }\end{array}$ & $\begin{array}{c}\phi_{\mathbf{c}} \\
\text { (degrees) }\end{array}$ & $\begin{array}{c}\phi_{\mathbf{p}} \\
\text { (degrees) }\end{array}$ & $\begin{array}{c}\text { Cohesive } \\
\text { intercept } \\
\text { (from best } \\
\text { fit line), } \\
\text { (kPa) }\end{array}$ & $\begin{array}{c}\text { Corresponding } \\
\phi \text { from best fit } \\
\text { line }\end{array}$ \\
\hline $20: 80$ & $\sim 50: 50$ & 30.1 & 31.0 & 14.5 & 27.1 \\
\hline $25: 75$ & $\sim 60: 40$ & 30.6 & 31.1 & 17.5 & 28.0 \\
\hline $35: 75$ & $\sim 70: 30$ & 32.0 & 32.0 & 10.3 & 29.6 \\
\hline
\end{tabular}




\subsection{Pullout Response}

The pullout response of various reinforcements embedded in tire shred-sand mixtures of varying mixing ratio is presented here. The pullout response was determined by conducting laboratory pullout tests on reinforcement ladders (metal straps), metal strips and geogrids embedded in tire shred-sand mixtures. The dimensions of the sample were $1.0 \mathrm{~m} \times 0.38 \mathrm{~m} \times 0.40 \mathrm{~m}$ and the effective length of the reinforcement within the box was $0.74 \mathrm{~m}$. The reinforcement was embedded at a depth of $0.2 \mathrm{~m}$ from the top of the sample.

\subsubsection{Reinforcement Ladders (or Metal Straps)}

Figure 1.3 shows the photograph of the reinforcement ladder (metal strap) used for pullout testing that was manufactured by the Reinforced Earth Company. Figures 1.4 and 1.5 show the plan and sectional view of the reinforcement ladder. Table 1.7 shows the summary of results of the pullout testing conducted on reinforcement ladders embedded in tire shred-sand mixtures at different mixing ratios. Using the results, equations were fitted to determine the pullout resistance factor, $\mathrm{F}^{*}$ at different depths. Table 1.8 shows the fitted equations to determine $\mathrm{F}^{*}$ at different depths of the wall. The results can be used with application of minimal caution. 


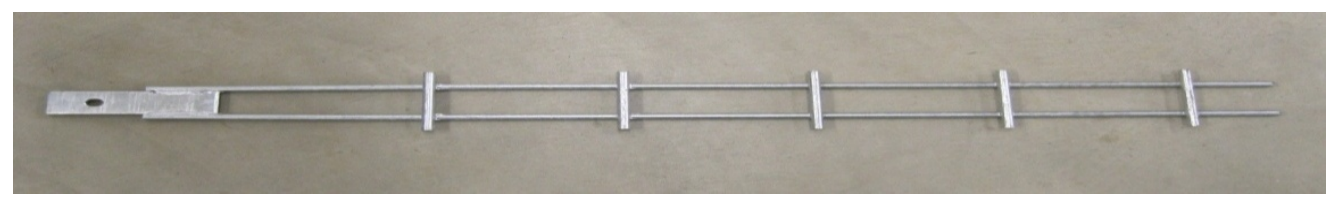

Figure 1.3 Photograph of reinforcement ladder manufactured by The Reinforced Earth Company (metal strap)

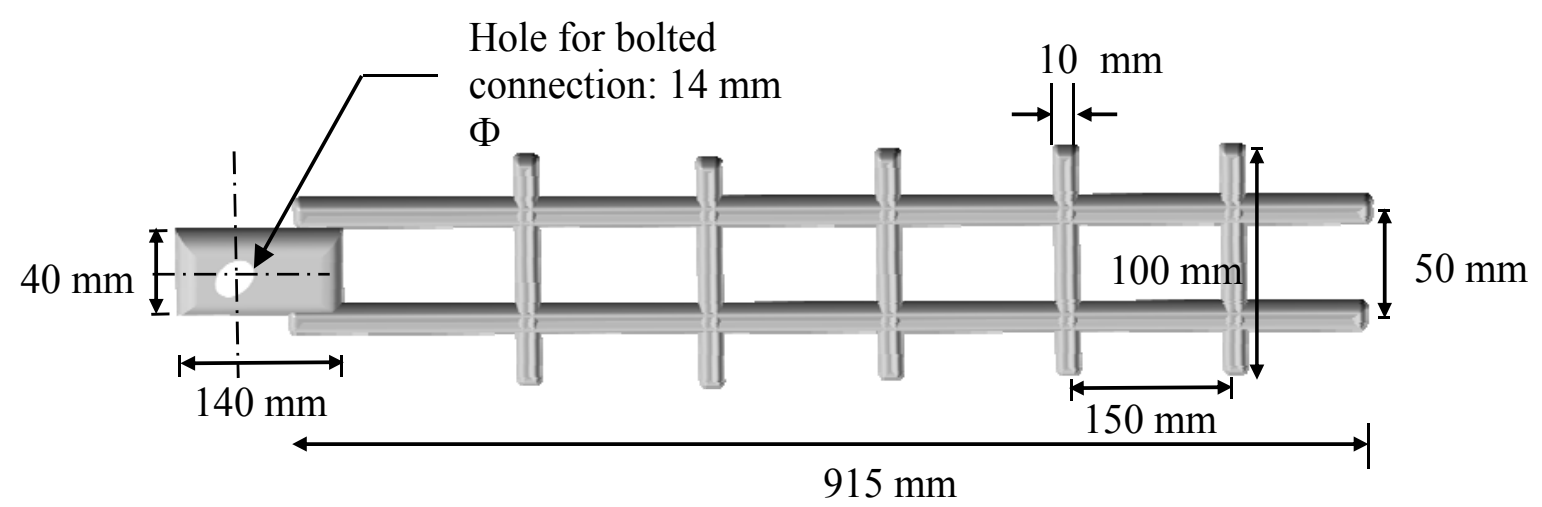

Figure 1.4 Reinforcement ladder - plan view

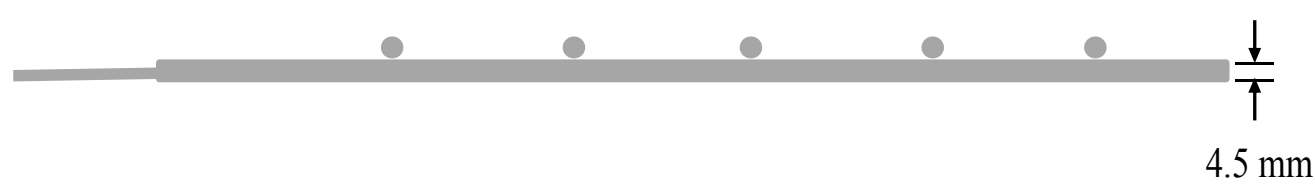

(c)

Figure 1.5 Reinforcement ladder - sectional view of the reinforcement ladder 
Table 1.7 Summary of pullout test results conducted on reinforcement ladders (metal straps) embedded in tire shred-sand mixtures ( 2 to 4 inches tire shreds + Ottawa sand)

\begin{tabular}{|c|c|c|c|c|c|c|c|c|}
\hline $\begin{array}{c}\text { Tire } \\
\text { shred- } \\
\text { Sand } \\
\text { mixing } \\
\text { ratio (by } \\
\text { volume) }\end{array}$ & $\begin{array}{c}\text { Tire } \\
\text { shred- } \\
\text { Sand ratio } \\
\text { (by } \\
\text { weight) }\end{array}$ & $\begin{array}{l}\text { Confining } \\
\text { pressure } \\
(\mathrm{kPa})\end{array}$ & $\begin{array}{c}\text { Maximum } \\
\text { dry unit } \\
\text { weight } \\
\left(\mathrm{kN} / \mathrm{m}^{3}\right) \\
(\text { Yoon } \\
2006)\end{array}$ & $\begin{array}{l}\text { Average } \\
\text { dry unit } \\
\text { weight in } \\
\text { pullout } \\
\text { box } \\
\left(\mathrm{kN} / \mathrm{m}^{3}\right)\end{array}$ & $\begin{array}{c}\text { Average } \\
\text { relative } \\
\text { compaction } \\
(\%)\end{array}$ & $\begin{array}{c}\text { Average } \\
\text { sand-matrix } \\
\text { unit weight } \\
\left(\mathrm{kN} / \mathrm{m}^{3}\right)\end{array}$ & $\begin{array}{l}\text { Reinforcement } \\
\text { ladder (metal } \\
\text { strap) pullout } \\
\text { capacity }(\mathrm{kN})\end{array}$ & $\begin{array}{l}\text { Pullout } \\
\text { resistance } \\
\text { factor, F* }\end{array}$ \\
\hline \multirow{3}{*}{$0: 100$} & \multirow{3}{*}{$0: 100$} & 40 & \multirow{3}{*}{18.0} & \multirow{3}{*}{17.2} & \multirow{3}{*}{95.6} & \multirow{3}{*}{17.2} & 4.2 & 1.31 \\
\hline & & 65 & & & & & 4.9 & 0.97 \\
\hline & & 90 & & & & & 6.6 & 0.95 \\
\hline \multirow{3}{*}{$50: 50$} & \multirow{3}{*}{$20: 80$} & 40 & \multirow{3}{*}{17.1} & \multirow{3}{*}{15.6} & \multirow{3}{*}{91.2} & \multirow{3}{*}{16.7} & 6.1 & 1.91 \\
\hline & & 65 & & & & & 7.2 & 1.43 \\
\hline & & 90 & & & & & 8.3 & 1.20 \\
\hline \multirow{3}{*}{$60: 40$} & \multirow{3}{*}{$25: 75$} & 40 & \multirow{3}{*}{16.6} & \multirow{3}{*}{14.8} & \multirow{3}{*}{89.1} & \multirow{3}{*}{15.8} & 5.5 & 1.73 \\
\hline & & 65 & & & & & 8.0 & 1.59 \\
\hline & & 90 & & & & & 9.5 & 1.38 \\
\hline \multirow{3}{*}{$70: 30$} & \multirow{3}{*}{$35: 65$} & 40 & \multirow{3}{*}{14.2} & \multirow{3}{*}{12.3} & \multirow{3}{*}{86.5} & \multirow{3}{*}{12.3} & 6.4 & 2.04 \\
\hline & & 65 & & & & & 9.4 & 1.88 \\
\hline & & 90 & & & & & 9.7 & 1.42 \\
\hline
\end{tabular}


Table 1.8 Equations fitted for determining $\mathrm{F}^{*}$ at any depth for reinforcement ladders embedded in tire shred-sand mixtures

TS : sand mixing ratio (by weight)
Equation fitted between depth $(\mathrm{z})$ and $\mathrm{F}^{*}$
Coefficient of determination, $\mathbf{R}^{2}$

$20: 80$

$$
\begin{gathered}
=-(\mathrm{z}-9.9995) / 3.957 \text { for } 0 \leq \mathrm{z}<7.7 \mathrm{~m} \\
=\tan (\quad) \text { for } \mathrm{z} \geq 7.7 \mathrm{~m}
\end{gathered}
$$

$25: 75$

$$
\begin{gathered}
=-(\mathrm{z}-17.457) / 8.512 \text { for } 0 \leq \mathrm{z}<12.32 \mathrm{~m} \\
=\tan (\quad) \text { for } \mathrm{z} \geq 12.32 \mathrm{~m}
\end{gathered}
$$

$$
\begin{gathered}
=-(\mathrm{z}-14.108) / 5.244 \text { for } 0 \leq \mathrm{z}<12.32 \mathrm{~m} \\
=\tan (\quad) \text { for } \mathrm{z} \geq 10.83 \mathrm{~m}
\end{gathered}
$$

\subsubsection{Ribbed-Metal Strip}

Figure 1.6 shows the photograph of the ribbed-metal strip used for pullout testing.

Figures 1.7 and 1.8 show the plan and sectional view of the ribbed-metal strip manufactured by The Reinforced Earth Company. Table 1.9 shows the summary of results of the pullout testing conducted on ribbed-metal strips embedded in tire shredsand mixtures prepared using various sizes of tires and Ottawa sand. Using the results, equations can be fitted to determine the pullout resistance factor, $\mathrm{F}^{*}$ at different depths, as shown in the case of reinforcement ladders. 


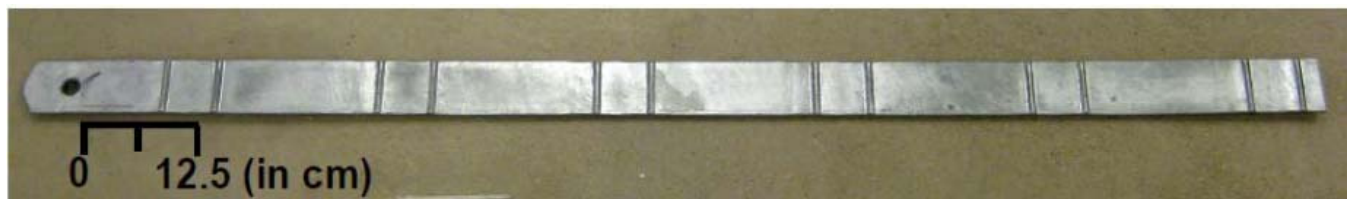

Figure 1.6 Photograph of ribbed-metal strip manufactured by The Reinforced Earth Company

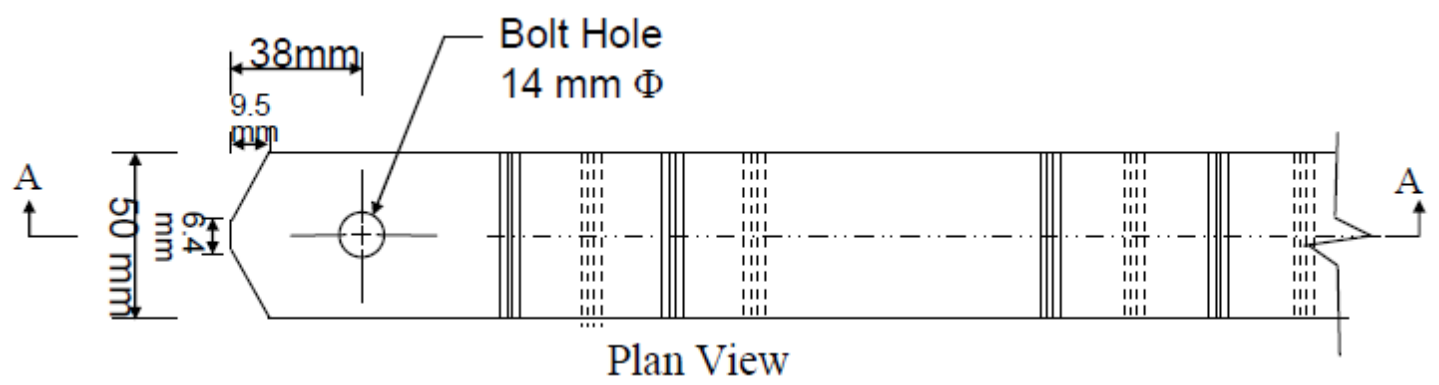

Figure 1.7 Plan view of ribbed-metal strip

4 mn एँ

Figure 1.8 Sectional view of ribbed-metal strip 
Table 1.9 Summary of pullout tests conducted on ribbed-metal strips embedded in tire shred-sand mixtures (varying size of tires + Ottawa sand)

\begin{tabular}{|c|c|c|c|c|c|c|c|c|}
\hline $\begin{array}{l}\text { Size of } \\
\text { tire } \\
\text { pieces }\end{array}$ & $\begin{array}{c}\text { Tire } \\
\text { shred- } \\
\text { sand ratio } \\
\text { (by } \\
\text { weight) }\end{array}$ & $\begin{array}{l}\text { Tire shred- } \\
\text { sand ratio } \\
\text { (by } \\
\text { volume) }\end{array}$ & $\begin{array}{c}\text { Confining } \\
\text { pressure } \\
(\mathrm{kPa})\end{array}$ & $\begin{array}{c}\text { Maximum } \\
\text { dry unit } \\
\text { weight } \\
\left(\mathrm{kN} / \mathrm{m}^{3}\right) \\
(\text { Yoon } 2006)\end{array}$ & $\begin{array}{c}\text { Average } \\
\text { dry unit } \\
\text { weight in } \\
\text { pullout box }\end{array}$ & $\begin{array}{c}\text { Relative } \\
\text { compaction } \\
(\%)\end{array}$ & $\begin{array}{l}\text { Ribbed } \\
\text { metal strip } \\
\text { pullout } \\
\text { capacity } \\
(\mathrm{kN})\end{array}$ & $\begin{array}{l}\text { Pullout } \\
\text { resistance } \\
\text { factor, } \\
\mathrm{F}^{*}\end{array}$ \\
\hline $\begin{array}{c}\text { Ottawa } \\
\text { Sand }\end{array}$ & $0: 100$ & $0: 100$ & $\begin{array}{l}40 \\
65 \\
90\end{array}$ & 18.0 & 17.2 & 95.5 & $\begin{array}{l}4.4 \\
5.2 \\
5.8\end{array}$ & $\begin{array}{l}1.49 \\
1.08 \\
0.87\end{array}$ \\
\hline $\begin{array}{c}9.5 \mathrm{~mm} \\
\text { size } \\
\text { chips } \\
\end{array}$ & $25: 75$ & $50: 50$ & $\begin{array}{l}40 \\
65 \\
90 \\
\end{array}$ & 15.2 & 14.3 & 94.3 & $\begin{array}{l}3.2 \\
4.0 \\
4.9 \\
\end{array}$ & $\begin{array}{l}1.08 \\
0.83 \\
0.74 \\
\end{array}$ \\
\hline $\begin{array}{c}50- \\
100 \mathrm{~mm}\end{array}$ & $25: 75$ & $58: 42$ & $\begin{array}{l}40 \\
65 \\
90 \\
\end{array}$ & 16.6 & 14.7 & 88.5 & $\begin{array}{l}3.1 \\
3.7 \\
4.4 \\
\end{array}$ & $\begin{array}{l}1.05 \\
0.77 \\
0.66 \\
\end{array}$ \\
\hline $\begin{array}{c}100- \\
200 \mathrm{~mm}\end{array}$ & $25: 75$ & $69: 31$ & $\begin{array}{l}40 \\
65 \\
90\end{array}$ & 16.5 & 14.2 & 86.2 & $\begin{array}{l}3.0 \\
3.4 \\
3.8\end{array}$ & $\begin{array}{l}1.01 \\
0.71 \\
0.57\end{array}$ \\
\hline
\end{tabular}




\subsubsection{Geogrids}

Figure 1.9 shows the UX-1500 MSE geogrids used for pullout testing that were manufactured by Tensar. Table 1.10 provides the properties of the geogrids used for testing. Table 1.11 presents the summary of pullout test results conducted on geogrids embedded in tire shred-sand mixtures prepared with varying sizes of tires and Ottawa sand.

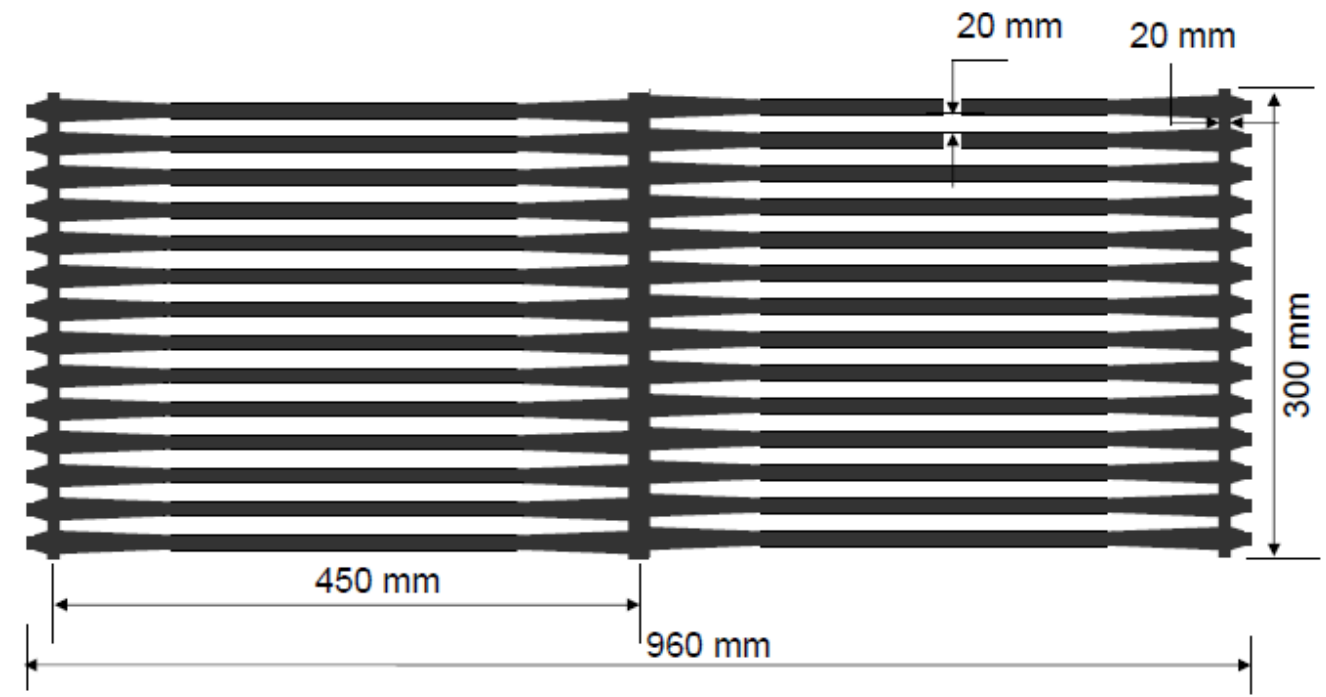

Figure 1.9 UX 1500 MSE geogrid manufactured by Tensar 
Table 1.10 Properties of geogrids used in pullout testing (UX 1500 MSE)

\begin{tabular}{ccc}
\hline Property & unit & Machine Direction (MD) Value \\
\hline True initial modulus & $\mathrm{kN} / \mathrm{m}$ & 1580 \\
Tensile strength at $5 \%$ strain & $\mathrm{kN} / \mathrm{m}$ & 52 \\
$\begin{array}{c}\text { Long-term allowable load in } \\
\text { sands, silts and clay } \\
\text { Long-term allowable load in well } \\
\text { graded sands } \\
\text { Long-term allowable load in } \\
\text { aggregate }\end{array}$ & $\mathrm{kN} / \mathrm{m} / \mathrm{m}$ & 45.2 \\
Junction strength & $\mathrm{kN} / \mathrm{m}$ & 43.9 \\
$\mathrm{kN} / \mathrm{m}$ & 43.1 \\
\hline
\end{tabular}

Table 1.11 Summary of results of pullout tests on geogrids embedded in the mixture

\begin{tabular}{cccc}
\hline $\begin{array}{c}\text { Size of } \\
\text { shredded tires }\end{array}$ & $\begin{array}{c}\text { Tire shred:sand } \\
\text { ratio (by weight) }\end{array}$ & $\begin{array}{c}\text { Confining } \\
\text { pressure }(\mathrm{kPa})\end{array}$ & $\begin{array}{c}\text { Pullout } \\
\text { capacity } \\
(\mathrm{kN} / \mathrm{m})\end{array}$ \\
\hline & $0: 100$ & 40 & 23.8 \\
Ottawa Sand & & 70 & 32.6 \\
& $25: 75$ & 100 & 42 \\
\hline 9.5mm & & 40 & 23.6 \\
nominal size & & 70 & 34.5 \\
& $25: 75$ & 100 & 40.1 \\
\hline 50-100mm in & & 40 & 19.7 \\
length & & 70 & 25.6 \\
& $25: 75$ & 100 & 30.7 \\
\hline 100-200mm & & 40 & 15.1 \\
in length & & 70 & 22.0 \\
& & 100 & 32.1 \\
\hline
\end{tabular}




\section{REFERENCES}

Balunaini (2009). "Experimental Study of the use of mixtures of sand and tire shreds as a geotechnical material", Ph.D. Thesis, School of Civil Engineering, Purdue University, West Lafayette, Indiana.

Berg, R.R., C.R. Barry, N.C.Samtani, (2009). "Design of mechanically stabilized earth walls and reinforced soils slopes - Volume I and II", FHWA, Report No. FHWA-NHI$10-024$.

Yoon, S. (2006). "Mechanical response of tire shred-sand mixtures and applications to geotechnical structures”, Ph. D. thesis, School of Civil Engineering, Purdue University, West Lafayette, Indiana. 